\title{
Polymerization Photoinitiators with Near-Resonance En- hanced Two-Photon Absorption Cross-Section: Towards High-Resolution Photoresists with Improved Sensitivity
}

\author{
Caroline Arnoux, ${ }^{a}$ Tatsuki Konishi, ${ }^{\text {b,c }}$ Emma Van Elslande, a Eric-Aimé Poutougnigni, a Jean- \\ Christophe Mulatier, a Lhoussain Khrouz, a Christophe Bucher, a Elise Dumont, a Kenji \\ Kamada, ${ }^{\text {b,c }}$ Chantal Andraud, a Patrice Baldeck, ${ }^{a}$ Akos Banyasz, a Cyrille Monnereau ${ }^{a}$ * \\ a Univ. Lyon, ENS Lyon, CNRS, Université Lyon 1, Laboratoire de Chimie, UMR 5182, 46 Allée d'Italie, 69364 \\ Lyon, France. Mail : cyrille.monnereau@ens-lyon.fr (corresponding author) \\ b Nanomaterials Research Institute (NMRI), National Institute of Advanced Industrial Science and Technology \\ (AIST), 1-8-31 Midorigaoka, Ikeda, Osaka, 563-8577 Japan \\ c Department of Chemistry, School of Science and Technology Kwansei Gakuin University, Sanda 669-1337, Japan
}

\begin{abstract}
We take advantage of the near-resonant enhancement of the third-order nonlinear response to engineer a two-photon polymerization photoinitiator with optimized efficiency in regard with literature benchmarks. We study in detail its linear and resonant third-order nonlinear optical properties, with a particular focus on photoinduced radical generation. Careful choice of peripheral substituents enable its direct solubilization into a mixture of commercial multifunctional acrylic monomers, resulting in homogeneous photoresist with good optical transparency upon simple mixing. When submitted to sub-nanosecond pulsed laser irradiation at $532 \mathrm{~nm}$, the resulting photoresist displays a polymerization threshold up to eight times lower than that obtained with classically used two-photon initiators, giving rise to sub-100 nm linewidth at $250 \mathrm{~nm}$ interline separation, and resulting in highly defined threedimensional (3D) structures.
\end{abstract}

\section{INTRODUCTION}

Within the last decade, the additive bulk manufacturing of 3D structures has become a major driving force in modern industry and is generally considered as one of the nine pillars of the so-called " $4^{\text {th }}$ industrial revolution", or industry $4.0 .^{1-2}$

Once limited to rapid prototyping of industrial high-value components and models, nowadays 3D printing has indeed become a relatively standard and affordable technology that is used in the manufacturing of many daily-life goods from plastic housing-shell to medical prostheses. ${ }^{3-}$ ${ }^{5}$ In the near future, 3D printing, owing to its unlimited design flexibility, is expected to substitute polymer thermoforming or milling in high-tonnage productions such as automotive and aerospace industries or the construction sector. . $^{6}$

As the range of applications that additive 3D manufacturing encompasses constantly expands, more and more demanding specific technical requirements and constraints tend to emerge..$^{9-10}$ In particular, fabrication of nano and micro-structured objects require resolution that are not accessible with standard layer-by-layer photolithography. ${ }^{11}$ -

${ }^{13}$ In that framework two-photon absorption-induced stereolithography is generally considered as a potential way to circumvent the limitations in achievable spatial resolution. ${ }^{14-15}$ However, the intrinsic characteristics of two-photon absorption, which allows localized polymerization reaction in the volume of the focal point of the laser beam, makes it very difficult to extend the polymerization to the fabrication of large structures with such high resolution. ${ }^{16-}$ 17

As a matter of fact, to become more than a "laboratory curiosity", two-photon additive manufacturing needs to face the challenge of high-throughput, large area printing of surfaces. ${ }^{17-24}$ To this end, endeavors are focused on the optimization of both optical setups ${ }^{16,25-26}$ and photoresist sensitivity.

From a chemical point of view, the design of an improved photoresist relies on a combination of a reactive monomer with high functionality, capable of generating an intricate polymer network even at low monomer conversion, ${ }^{27}$ and a highly sensitive photoinitiator $(\mathrm{PI}){ }^{28}$ Monomer sensitivity can be improved by removing inhibiting species such as molecular oxygen or exogenous inhibitors (such as $M E H Q$ ) generally included as stabilizers in monomer formulations. ${ }^{29}$ However, such strategies can severely impair the accessible resolution, as they favor radical diffusion outside of the irradiated volume, and result in a loss of control on voxel volume. ${ }^{30-31}$ Therefore, a majority of research works have been focused on improving PI efficiency. ${ }^{32-34}$ 
As a general statement, PI efficiency essentially relies on two parameters, namely $\mathrm{i} /$ the two-photon absorption cross-section ( $\left.\sigma_{T P A}\right)$, which quantifies the probability for the chromophore to undergo transition to its excited state upon laser irradiation through the simultaneous absorption of two photons, ii/ the radical generation quantum efficiency, which represents the probability for the excited molecule to generate a radical capable of initiating the reaction of polymerization. ${ }^{35}$

While a lot of recent researches have focused on the use of Pls with optimized radical generation quantum efficiency, ${ }^{34,}, 36-41$ the improvement of $\sigma_{\text {TPA }}$ has exclusively been addressed through a relatively classical chromophore engineering. ${ }^{42-50}$ This followed the Equation 1 that approximates in the so-called "three-level model" the imaginary part of the third-order hyperpolarizability $\mathrm{\gamma}$, responsible for the two-photon absorption phenomenon, where $\boldsymbol{P}$ denotes a permutation operator over the optical frequencies; $\mu_{i j}$, the transition dipole moment between the singlet state $i$ and the singlet state $j ; E_{i j}$, the transition energy; $\Delta \mu_{i j}$, the dipole moment difference between the $i$ and $j$ states and $\Gamma$ the associated damping factor; $\hbar \omega$, energy of the incident photon.

In all of these works, a general paradigm is that dipolar Pls should be selected so that their one-photon absorption (OPA) resonance wavelength lies closely to half of that of the operating laser ${ }^{51}$ By doing so, the sum of the energies of two incident photons is resonant with the energy gap between the ground and excited states of the PI (ie the $S_{1}$ state in the case of a dipole, and the $S_{2}$ state in the case of a quadrupole). Regarding the lowest TPA energy transition, this strategy leads to a minimization of the second term of the denominator for the $\mathrm{D}$ (dipolar molecules) or T (quadrupolar molecules) terms, thus to a maximization of the $y$ term. ${ }^{52}$

$$
\operatorname{Im} \gamma(-\omega ; \omega,-\omega, \omega)=\operatorname{Im} \boldsymbol{P}\left[\begin{array}{l}
+\frac{\mu_{01}^{2} \Delta \mu_{01}^{2}}{\left(E_{01}-\hbar \omega-i \Gamma\right)^{2}\left(E_{01}-2 \hbar \omega-i \Gamma\right)}(D) \\
+\frac{\mu_{01}^{2} \mu_{12}^{2}}{\left(E_{01}-\hbar \omega-i \Gamma\right)^{2}\left(E_{02}-2 \hbar \omega-i \Gamma\right)}(T) \\
-\frac{\mu_{01}^{4}}{\left(E_{01}-\hbar \omega-i \Gamma\right)^{2}\left(E_{01}+\hbar \omega+i \Gamma\right)}(N)
\end{array}\right]
$$

However, another way of maximizing y consists in minimizing the denominator terms by using an incident photon that comes close to the OPA resonance $\hbar \omega \approx E_{01}$ yet does not overlap with the linear absorption band

This phenomenon is known as the near-resonance enhancement of the $\sigma_{T P A},{ }^{53-55}$ and has been shown to play a significant role in the outstanding $\sigma_{\text {TPA }}$ values obtained for molecules such as extended porphyrin ${ }^{56}$ or squaraine ${ }^{57}$ derivatives mainly used in two-photon photodynamic therapy. ${ }^{58}$ To the best of our knowledge, only one example has been explicitly reported to date, that concerns twophoton induced microfabrication using cationic polymerization. ${ }^{59}$

Practically, working with a $532 \mathrm{~nm}$ pulsed laser, a blue absorbing chromophore structurally optimized following the general principles and guidelines of chromophores design for nonlinear optics (large $\pi$-conjugated structure, presence of electron donating and/or withdrawing groups) can be used as a near-resonant two-photon photosensitizer.

In this paper, we describe the synthesis and detailed characterizations of two such chromophores, using a common set of monomeric building blocks. These chromophores, named V-Shape and Oct, consist respectively in two and three branched oligo(phenyleneethynylene) derivatives, end terminated with aniline moieties used as strong electron donors. We investigate in detail their linear and nonlinear spectroscopic features, and study their mechanisms of decomposition upon photoirradiation. By comparison with commercially available benchmark Norrish I (Irgacure OXE2) and II (BDEBP and ITX) type of PIs (as shown in Figure 1), which have been previously used in two-photon microfabrication, ${ }^{60-65}$ we identify the nature of the photogenerated radicals and propose a mechanism accounting for their formation.
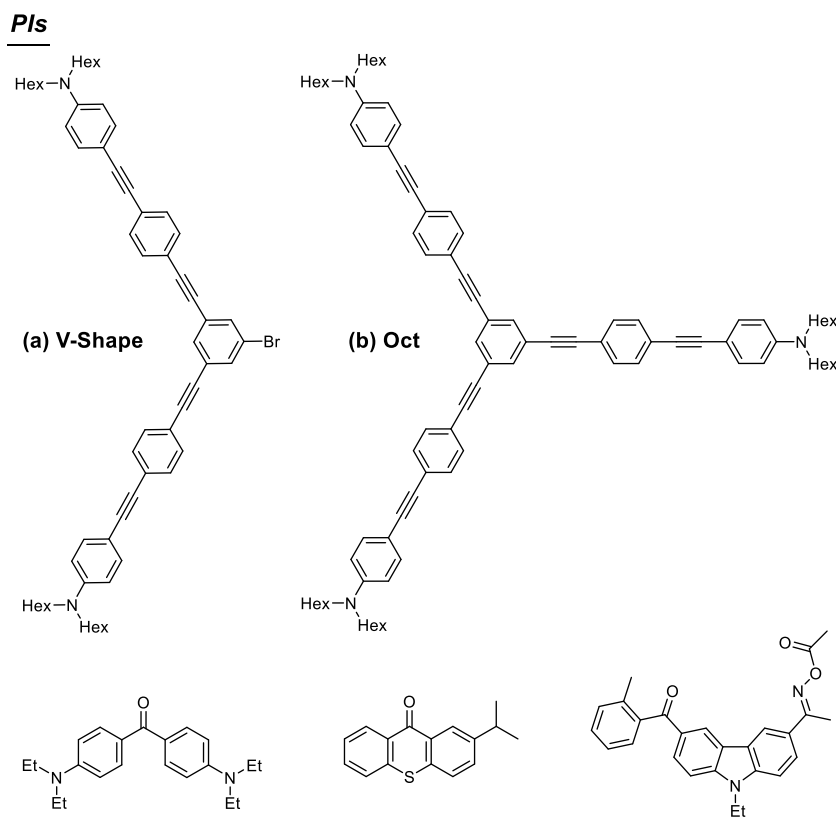

(c) BDEBP

(d) ITX

(e) OXE2

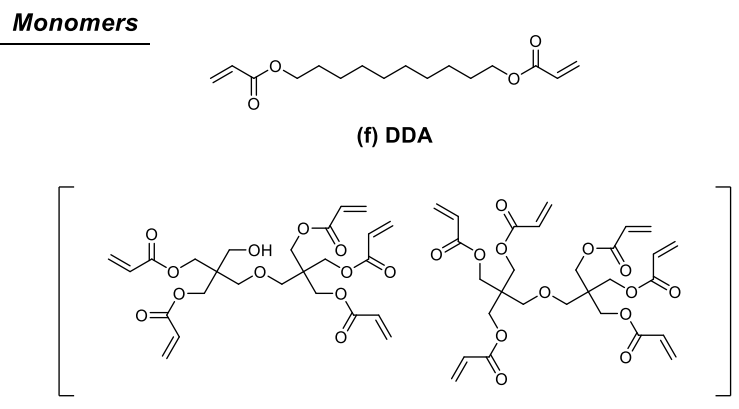

(g) DPPHA

Figure 1. Chemical structures of the five Pls investigated in this study and the two monomers that were used: (a) $\boldsymbol{V}$ Shape; (b) Oct; (c) 4,4'-Bis(diethylamino)benzophenone also called Michler's ethyl ketone BDEBP; (d) ITX; (e) OXE2 (f) 1,10-decanediol diacrylate $D D A$; (g) dipentaerythritol penta/hexa-acrylate DPPHA. 
Through a combined spectroscopic and electrochemical study, we study in details the mechanisms of radical generation and polymerization initiation. Finally, we show that the two new PIs reported in the study, when incorporated in a mixture of multifunctional acrylic monomers, provide significant benefits in terms of polymerization sensitivity with a polymerization threshold lowered up to eight times in comparison with archetypical Norrish I (OXE2, acyloxime ester) or Norrish II (Michler's ketone derivative, 4,4'bis(diethylamino)benzophenone BDEBP and isopropyl thioxanthone ITX) PIs similar to those used in recent works dealing with two-photon microfabrication, while maintaining high-resolution features.

\section{EXPERIMENTAL SECTION}

Materials and general methods. All reactions were performed under argon. Solvents were used without further purification. All reagents, including reactant 2 were purchased from Sigma Aldrich, TCl or Alfa Aesar and were used without further purification. The reaction progresses were monitored by thin layer chromatography (TCL) with silica gel 60 F254 using UV lamp detection. Column chromatography was performed using Acros Organics $(0.035-0.70 \mathrm{~mm})$ silicagel for purification. Dipentaerythritol penta-/hexa-acrylate (DPPHA) was purchased from Sigma Aldrich and 1,10-decanediol diacrylate (DDA) from TCl. 4,4'-bis(diethylamino)benzophenone also called Michler's ethyl ketone BDEBP was purchased from Merck and OXE2 from Angene Chemical. ${ }^{1} \mathrm{H}$ and ${ }^{13} \mathrm{C}$ NMR spectra in $\mathrm{CDCl}_{3}$ were recorded at room temperature on a BRUKER Avance spectrometer operating at 400 $\mathrm{MHz}$ for ${ }^{1} \mathrm{H}$ and $75 \mathrm{MHz}$ for ${ }^{13} \mathrm{C}$ respectively. Chemical shifts are reported in parts per million $(\delta, p p m)$ relatively to tetramethylsilane, using residual solvent peaks as internal standards $\left(\mathrm{CHCl}_{3}{ }^{1} \mathrm{H}: 7.26 \mathrm{ppm},{ }^{13} \mathrm{C}: 77.36 \mathrm{ppm}\right)$. Spectra are provided in supporting information (see Figures S1 and S2). High resolution mass spectrometry measurements were performed at Centre Régional de Mesures Physiques de l'Ouest (Université de Rennes, Rennes, France).

Synthesis. Synthesis of compounds 1, 3 and $\mathbf{4}$ have been already reported in the literature (

). ${ }^{66-67}$

V-Shape: 1 (465 mg, $1.21 \mathrm{mmol}$ ) and 2 (114 mg, 0.36 $\mathrm{mmol})$ were dissolved in a mixture of THF $(20 \mathrm{~mL})$ and $\mathrm{Et}_{3} \mathrm{~N}(15 \mathrm{~mL})$. The solution was degassed by argon bubbling for 1 hour. Then $\mathrm{Pd}\left(\mathrm{PPh}_{3}\right)_{2} \mathrm{Cl}_{2}(138 \mathrm{mg}, 5.4 \%)$ and Cul (69 mg, 10\%) were added. The reaction mixture was stirred at $90^{\circ} \mathrm{C}$ under argon for 48 hours. Solvents were removed under reduced pressure and the crude product was dissolved in dichloromethane. The solution was washed with $\mathrm{NH}_{4} \mathrm{Cl}$, water and pre-dried with saturated $\mathrm{NaCl}$ solution. The solution was dried with $\mathrm{Na}_{2} \mathrm{SO}_{4}$, filtered and solvents were remove under vacuum pressure. The product was then purified on silica gel with a mixture $\mathrm{PE} / \mathrm{CH}_{2} \mathrm{Cl}_{2}$ as eluent (from $9 / 1$ to $8 / 2$, v/v. solid deposition of the product) to give a brown oil (195 mg, 58\%). ${ }^{1} \mathrm{H}$ NMR $\left(400 \mathrm{MHz}, \mathrm{CDCl}_{3}\right): \delta 7.65$ (d, $\left.J=1.4 \mathrm{~Hz}, 2 \mathrm{H}\right), 7.64(\mathrm{t}, J=$ $1.3 \mathrm{~Hz}, 1 \mathrm{H}), 7.49(\mathrm{~s}, 8 \mathrm{H}), 7.39(\mathrm{~d}, J=8.9 \mathrm{~Hz}, 4 \mathrm{H}), 6.60(\mathrm{~d}$, $J=9.0 \mathrm{~Hz}, 4 \mathrm{H}), 3.30(\mathrm{t}, J=8.1 \mathrm{~Hz}, 6 \mathrm{H}), 1.61(\mathrm{t}, J=7.0$ $\mathrm{Hz}, 8 \mathrm{H}), 1.36-1.33(\mathrm{~m}, 24 \mathrm{H}), 0.93(\mathrm{t}, J=6.7 \mathrm{~Hz}, 12 \mathrm{H})$. ${ }^{13} \mathrm{C}$ NMR $\left(75 \mathrm{MHz}, \mathrm{CDCl}_{3}\right): \delta 148.2,133.9,133.0,132.3$, 131.6, 131.2, 125.3, 124.9, 122.0, 121.1, 111.2, 108.2, 93.5, 91.3, 88.5, 87.0, 51.0, 31.7, 27.2, 26.8, 22.7, 14.1. HRMS (ESI) $\mathrm{m} / \mathrm{z}$ calcd for $\left[\mathrm{C}_{62} \mathrm{H}_{71} \mathrm{BrN}_{2}+\mathrm{H}\right]^{+}$at 923.48734 , found 923.4871 .

Oct: 3 (700 mg, $1.44 \mathrm{mmol})$ and $4(65 \mathrm{mg}, 0.43 \mathrm{mmol})$ were dissolved in a mixture of THF $(15 \mathrm{~mL})$ and $\mathrm{Et}_{3} \mathrm{~N}(10$ $\mathrm{mL}$ ). The solution was degassed by argon bubbling for 1 hour. Then $\mathrm{Pd}\left(\mathrm{PPh}_{3}\right)_{2} \mathrm{Cl}_{2}(150 \mathrm{mg}, 5 \%)$ and $\mathrm{Cul}(80 \mathrm{mg}$, $9.6 \%$ ) were added. The reaction mixture was stirred at $70^{\circ} \mathrm{C}$ under argon for 24 hours. Solvents were removed under reduced pressure and the crude product was dissolved in dichloromethane. The solution was washed with $\mathrm{NH}_{4} \mathrm{Cl}$, water and pre-dried with saturated $\mathrm{NaCl}$ solution. The solution was dried with $\mathrm{Na}_{2} \mathrm{SO}_{4}$, filtered and solvents were remove under vacuum pressure. The product was then purified on silica gel with a mixture $\mathrm{PE} / \mathrm{CH}_{2} \mathrm{Cl}_{2}$ as eluent (from $9 / 1$ to $8 / 2$, v/v. solid deposition) to give a yellow oil (150 mg, 28\%). ${ }^{1} \mathrm{H}$ NMR (400 MHz, $\left.\mathrm{CDCl}_{3}\right): \delta 7.64$ (s, $3 \mathrm{H}), 7.48(\mathrm{~s}, 12 \mathrm{H}), 7.37(\mathrm{~d}, J=8.8 \mathrm{~Hz}, 6 \mathrm{H}), 6.58(\mathrm{~d}, J=$ $9.0 \mathrm{~Hz}, 6 \mathrm{H}), 3.28(\mathrm{t}, J=7.4 \mathrm{~Hz}, 12 \mathrm{H}), 1.59(\mathrm{t}, J=7.7 \mathrm{~Hz}$, $12 \mathrm{H}), 1.41-1.27(\mathrm{~m}, 36 \mathrm{H}), 0.91(\mathrm{t}, J=6.6 \mathrm{~Hz}, 18 \mathrm{H}) .{ }^{13} \mathrm{C}$ NMR $\left(75 \mathrm{MHz}, \mathrm{CDCl}_{3}\right): \delta 148.1,134.0,133.0,131.6$, 131.2, 124.7, 124.1, 121.4, 111.2, 108.3, 93.4, 90.6, 89.2, 87.0, 51.0, 31.7, 27.2, 26.8, 22.7, 14.1. HRMS (ESI) m/z calcd for $\left[\mathrm{C}_{90} \mathrm{H}_{105} \mathrm{~N}_{3}+\mathrm{H}\right]{ }^{+}$at 1228.83813 , found 1228.8375 .

Absorption and luminescence. Absorption spectra were recorded on a JASCO V-650 spectrometer in dilute dichloromethane solution (about $10^{-6} \mathrm{~mol} / \mathrm{L}$ ). Emission spectra were measured using a Horiba-Jobin-Yvon Fluorolog-3 spectrofluorimeter equipped with a three-slit double grating excitation and emission monochromator with a dispersion of $2.1 \mathrm{~nm} / \mathrm{mm}$ (1200 groove $/ \mathrm{mm}$ ). A R928 detector was used. Corrections

were applied for both the excitation light intensity variation (lamp and grating) and the emission spectral response (detector and grating). Absolute fluorescence quantum yields were measured in diluted dichloromethane solutions using a GMP G8 integrating sphere. Sample were introduced into a capillary quartz tube placed into the cavity of the sphere. Differences between the peak intensities of the lamp and the emission profiles were minimized using a density filter $(0.5 \%)$ to attenuate the lamp signal and thus ensure that the collected signal remained in the linear range of the detector.

Z-Scan TPA measurement. Z-scan measurements were conducted in dichloromethane (spectroscopic grade) solutions for V-Shape $(0.61 \mathrm{mM})$, Oct $(1.52 \mathrm{mM})$ and BDEBP $(9.8 \mathrm{mM})$. The open aperture $z$-scan method ${ }^{68}$ was employed for all samples held in 2-mm quartz cuvette. 


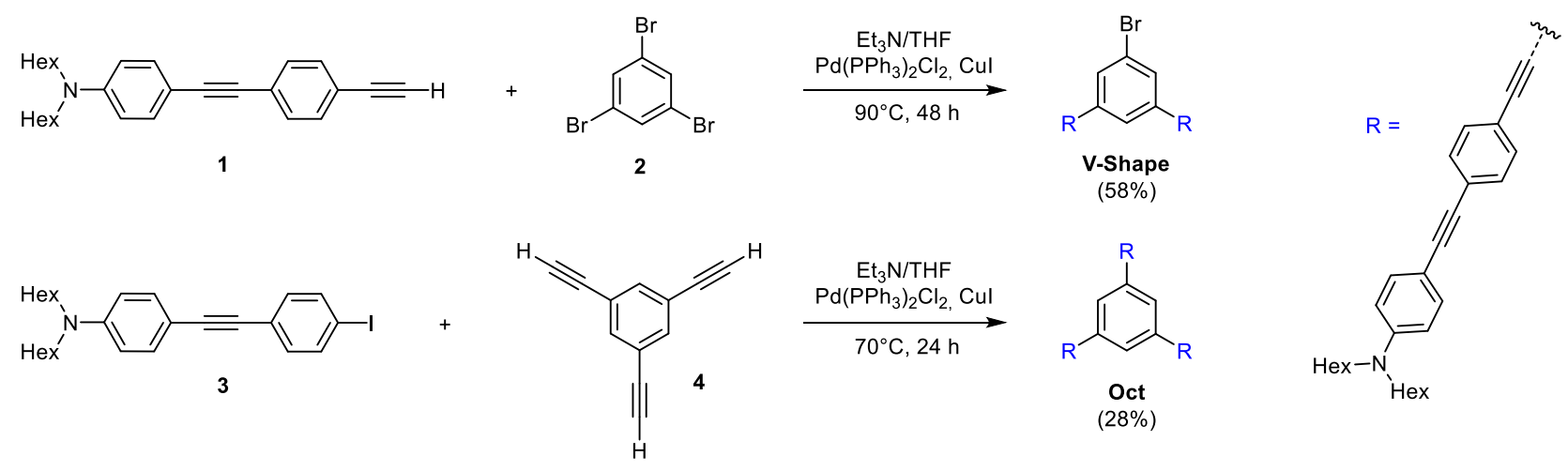

Scheme 1. Synthesis of target compounds V-Shape and Oct.

Two measurement procedures were used: (1) wavelength (WL)-scan measurement, where the single-power measurement was repeated for difference excitation wavelengths and the z-scan traces were analyzed by assuming TPA process; and (2) power-scan measurement, where measurements were repeated at least with four different incident powers for each excitation wavelength to confirm that the observed nonlinear absorption is dominated by TPA. The combination of these measurement procedures allows us to obtain fast and reliable results because the WL-scan results show the overview of the spectra and the power-scan results for some selected wavelengths make sure that the observed results are caused by TPA. The incident powers (excitation intensities) used differed depending on the wavelength range of the laser i.e. the configuration of the femtosecond optical parametric amplifier Spectra-Physics TOPAS Prime and were $0.40 \mathrm{~mW}$ $\left(80 \sim 100 \mathrm{GW} / \mathrm{cm}^{2}\right)$ for $600 \sim 900 \mathrm{~nm}$ range (SHS, SHI) and $0.25 \mathrm{~mW}\left(60 \sim 170 \mathrm{GW} / \mathrm{cm}^{2}\right)$ for $485 \sim 600 \mathrm{~nm}$ range (SFS, SFI) for the WL-scan. On the other hand, power-scan was made for these powers and three weaker powers (factors of $0.7,0.5$ and 0.3 ). The typical pulse width and the Rayleigh range were 90 120 fs and 6 11 mm although they changed depending on the wavelength. MPPBT/DMSO 68 and bis-MSB /CLF ${ }^{69}$ were used as reference sample for 600-900 nm and 485-600 nm respectively.

EPR-Spin Trapping (ST). Dichloromethane solutions of each $\mathrm{PI}$ in presence of trapping agent (N-tert-Butyl-a-phenylnitrone PBN, and 5,5-Dimethyl-1-pyrroline $\mathrm{N}$-oxide DMPO) were carefully degassed through five freezepomp-thaw cycles in quartz EPR tubes. The spectra of the irradiated samples (M365L2 Thorlabs LED, 365nm) were recorded in situ. EPR assays were all carried out at room temperature using a Bruker E500 spectrometer operating at X-band (9.33-9.87 GHz), standard cavity, with $100 \mathrm{KHz}$ modulation frequency. The instrument settings were as the following: microwave power: $0.2-64 \mathrm{~mW}$; modulation amplitude: $1 \mathrm{G}$. Hyperfine coupling constants a values were obtained with simulation of experimental spectra using Easyspin (Matlab toolbox) and compared with the literature.

Cyclic Voltamperometry. Cyclic voltammetry (CV) curves have been recorded using a SP300 Biologic potentiostat. All studies were conducted under an argon at- mosphere in a standard one-compartment three-electrode electrochemical cell. An automatic ohmic drop compensation procedure was systematically performed when using CV. Vitreous carbon $(\varnothing=3 \mathrm{~mm})$ working electrodes ( $\mathrm{CH}$ Instruments) were polished with $2 \mu \mathrm{m}$ diamond paste (PRESI SA) before each recording. $\mathrm{Ag} / \mathrm{AgNO}_{3}(\mathrm{CH}$ Instruments, $10^{-2} \mathrm{~mol} / \mathrm{L}+$ TBAP $10^{-1} \mathrm{~mol} / \mathrm{L}$ in $\mathrm{CH}_{3} \mathrm{CN}$ ) was used as a reference electrode and bis $\left(\eta^{5}\right.$-cyclopentadienyl)iron (ferrocene) was used as an internal reference. Dichloromethane $(99.9 \%$, extra-dry, stabilized with amylene) was purchased from Acros and used as received. Tetra- $n$-butylammonium perchlorate (TBAP, Fluka puriss.) was purchased and dried under vacuum at $60^{\circ} \mathrm{C}$ for $24 \mathrm{~h}$ before use.

DFT calculations. Density functional theory (DFT) calculations were performed with the Gaussian 16 Rev B.01 suite of programs. ${ }^{70}$ Structures of V-Shape in the neutral, radical cationic and radical anionic states were computed at the M06HF/6-31G(d,p) level of theory, both in gas phase and in dichloromethane to estimate electronic energies as well as redox potentials. Absolute redox potentials were estimated at $298 \mathrm{~K}$, after frequencies calculations according to the computational protocol proposed by Truhlar and coworkers. ${ }^{71}$

Stern-Volmer experiments. Emission spectra of the tested PIs in dichloromethane were measured using a Horiba-Jobin Yvon Fluorolog-3 spectrofluorimeter from 375 to $700 \mathrm{~nm}$ at room temperature with an excitation at $\lambda_{\mathrm{ex}}=370 \mathrm{~nm}$. A concentrated solution of DPPHA at isomolar concentration of $\mathrm{PI}$ as in the first solution was then added until reaching the final concentration of DPPHA. No change was observed, indicating an absence of electron or energy transfer between the singlet excited state of VShape or Oct and the monomer.

Photolysis studies. Photolysis studies were performed in a home-made setup consisting of a $365 \mathrm{~nm}$ Thorlabs LED (M365L2) equipped with a convex lens (plano-convex $f=25.4 \mathrm{~mm}$, Thorlabs) aiming to obtain a beam diameter of about $9 \mathrm{~mm}$ through the sample cuvette $(10 \times 10 \mathrm{~mm})$ placed $10 \mathrm{~cm}$ downstream of the LED. LED power was measured on a thermal power sensor (S405C, Thorlabs) placed at the same distance. Prior to the study, dichloromethane solutions of $\mathrm{PI}$ in presence or absence of monomer were carefully degassed through five freezepomp-thaw cycles and were stirred during photolysis. 
Microfabrication. Photoresist preparation: the PIs were mixed with DDA and stirred for 30 min at room temperature with additional dichloromethane when needed. No solvent was necessary for V-Shape and Oct solubilization. DPPHA was then added. The solution was stirred mechanically for $1 \mathrm{~min}$ and then magnetically for $30 \mathrm{~min}$. Good homogeneity was obtained without filtration, ensuring that the desired concentration was achieved in each photoresists tested.

Fabrication and setup: the 3D microfabrication was carried out using a Microlight3D printer $\mu$ FAB-3D based on a Zeiss Axiovert 200 inverted microscope equipped with a $X Y Z$ piezo nanomanipulator allowing the translation of the sample relative to the laser focal point, and a CMOS camera mounted behind a dichroic mirror for monitoring of the polymerization process. The laser module includes a microchip self-Q-switched frequency-doubled Nd:YAG laser at $532 \mathrm{~nm}$ providing $560 \mathrm{ps}$ pulses at a repetition rate of $11.7 \mathrm{kHz}$ with a maximum average power of $11.5 \mathrm{~mW}$ at entrance of the objective. Average laser power were measured at the entrance pupil of the objective on a standard photodiode power sensor (S120VC, Thorlabs). The incident beam was focused with a high numerical aperture objective (x100, NA 1.40, oil immersion, Zeiss PlanAPOCHROMAT). The laser power was controlled by an acousto-optic modulator which was computer-piloted via LITHOS software as well as the displacement of the sample and the scanning speed. The sample consists of a drop of photoresist deposited on a borosilicate coverslip (170 $\mu \mathrm{m} \pm 5 \mu \mathrm{m}$ thick). After the manufacturing process, the structures were obtained by washing away the unreacted monomer with two successive 10-minute acetone baths.

Microstructure characterization: samples were metallized with $5 \mathrm{~nm}$ platinum via vacuum deposition with Leica EM ACE600 sputter coater to prevent charge accumulation and enhance the contrast during the following Scanning Electron Microscopy (SEM) observations. The line widths were measured using a top view and the line heights using a grazing view $\left(90^{\circ}\right)$ with a Zeiss Supra 55VP SEM. A home-made python program was used to automatically determine the edges of the lines and give an average value of a line width over a length of several micrometers of the middle of each line. More details are provided in supporting information. The error bars correspond to the $95 \%$ confidence interval given by the student's t-distribution. Thus, it provides information on the error resulting from the measurement of the line widths and heights by SEM. The values were considered without subtracting the thickness of the platinum layer (expected at twice $5 \mathrm{~nm}$ i.e. $10 \mathrm{~nm})$.

\section{RESULTS AND DISCUSSION}

Synthesis. V-Shape and Oct were synthesized using a general synthetic approach and building blocks depicted in previous articles. ${ }^{66-67}$ The final key-step of both syntheses consists in a Sonogashira coupling of respectively a tribromobenzene 2 with the extended alkyne terminated aniline $\mathbf{1}$, and three iodoalkyne chains 3 with a 1,3,5-triethynylbenzene 4 (see

), which synthesis has already been reported. ${ }^{66}$ Depending on the reaction conditions, it is possible to drive the reaction towards the almost exclusive formation of either V-Shape or Oct. The preferential formation of V-Shape rather than of Oct following the first pathway could be explained by the electron enrichment of the halide through the first two couplings with the alkyne-aniline moiety, which is detrimental to the oxidative addition step of $\mathrm{Pd}(0)$ into the remaining carbon-halide bond. Both target materials can be recovered in moderate yields after purification of the crude product on silica gel. Detailed synthetic protocols and characterizations are featured in the experimental section.

Spectroscopy. Main spectroscopic data for both studied molecules are gathered in Erreur! Source du renvoi introuvable., along with those of the studied benchmark PIs, when available. Both molecules are characterized by a broad and structureless absorption band (see Figure 2) that peaks at the limit between near UV and visible (390 $\mathrm{nm}$ ) and extends into the violet and blue range of the visible spectra. The shape of the spectrum in dichloromethane solvent, along with the characteristic large oscillator strength, is typical of an intramolecular charge transfer (ICT) band, characteristic of two-photon absorbing chromophores. In addition, a similarly broad fluorescence band is observed peaking at $508 \mathrm{~nm}$ and $495 \mathrm{~nm}$ for VShape and Oct respectively.

Table 1. Summary of the main photophysical and electrochemical parameters for both molecules and references in dichloromethane: maximum absorption wavelengths $\left(\lambda_{\text {max-abs}}\right)$, molar extinction coefficients $(\varepsilon)$ at $\lambda_{\text {max }}$ abs, maximum emission wavelengths $\left(\lambda_{\max -e m}\right)$, fluorescence quantum yields $\varphi_{i}$, cathodic peak potential $\left(E_{p c}\right)$, anodic peak potential $\left(E_{p a}\right)$, standard free energy changes for photoinduced electron transfer $\left(\Delta G_{E T}^{0}\right)$ and TPA cross-sections at $532 \mathrm{~nm}\left(\boldsymbol{\sigma}_{T P A}^{\mathbf{5 3 2 n m}}\right)$.

\begin{tabular}{ccccccccc}
\hline PI & $\begin{array}{c}\lambda_{\text {max-abs }} \\
(\mathrm{nm})\end{array}$ & $\varepsilon\left(10^{4} \mathrm{~L} / \mathrm{mol} / \mathrm{cm}\right)$ & $\begin{array}{c}\lambda_{\text {max-em }} \\
(\mathrm{nm})\end{array}$ & $\varphi_{\mathrm{f}}(\%)$ & $\mathrm{E}_{\mathrm{pc}}(\mathrm{V})$ & $\mathrm{E}_{\mathrm{pa}}(\mathrm{V})$ & $\begin{array}{c}\Delta \mathrm{G}_{E T}^{0} \\
(\mathrm{~kJ} / \mathrm{mol})\end{array}$ & $\sigma_{T P A}^{532 n m}(\mathrm{GM})$ \\
\hline V-Shape & 386 & 8.9 & 508 & 66 & -2.33 & +0.37 & -11.4 & $1500 \pm 200$ \\
Oct & 386 & 14.8 & 495 & 79 & -2.48 & +0.36 & -2.3 & $740 \pm 150$ \\
BDEBP & 362 & 4.1 & - & - & na & +0.49 & na & $77 \pm 11$ \\
ITX & $386^{\mathrm{a}}$ & $0.7 \mathrm{a}$ & $420^{\mathrm{a}}$ & $9 \mathrm{a}$ & na & na & na & na \\
OXE2 & 340 & 2.1 & 464 & $\approx 0$ & na & na & na & na \\
\hline
\end{tabular}


Peak potential values measured by CV for V-Shape, Oct and BDEBP (1 mmol/L) in the presence of tetra- $n$-butylammonium perchlorate $(0.1 \mathrm{~mol} / \mathrm{L}), E(\mathrm{~V}) v s . E_{\mathrm{ref}}\left[\mathrm{Fc} / \mathrm{Fc}^{+}\right]$, at a vitreous carbon working electrode $\emptyset=3 \mathrm{~mm}, 298 \mathrm{~K}, v=0.1 \mathrm{~V} / \mathrm{s}$. na: not available. a previously reported, ${ }^{72}$ in chloroform.

TPA spectra were measured for both compounds by the Z-scan technique, over a range of wavelengths covering the 500-1000 $\mathrm{nm}$ region. In both cases, in addition to the "regular" TPA band corresponding to the $\mathrm{S}_{0} \rightarrow \mathrm{S}_{1}$ transition (excitation to the lowest lying excited state, similar to that reached upon OPA at $390 \mathrm{~nm}$ ), another strong TPA transition is also seen, as expected, as the laser wavelength gets closer to the OPA resonance wavelength. Interestingly while OTPA peaks ca. $500 \mathrm{GM}$ for the regular TPA band, consistent with values usually found on related chromophore with similar $\pi$-conjugated architectures, ${ }^{66}$, 73-74 a much higher value is found at the near-resonant TPA band. In particular, at the operating wavelength of the frequency doubled Nd-YAG laser used in our microfabrication setup (532 nm), бTPA reaches a value about $1500 \pm 100 \mathrm{GM}$ for V-Shape, then increases up to a value ca. $2000 \mathrm{GM}$ at $500 \mathrm{~nm}$. The $532 \mathrm{~nm}$ value of Oct is significantly lower $(740 \pm 150 \mathrm{GM})$, because of a more energetic onset of the near-resonant transition, consistent with the slightly blue-shifted onset of the ICT band in the OPA spectrum, but reaches a similar value at $500 \mathrm{~nm}(2200$ GM). These very high $\sigma_{T P A}$ values are reminiscent of those found for expanded porphyrin and squaraine derivatives, for which near-resonant enhancement of TPA has been shown to play an important role in the nonlinear response. ${ }^{56-57,75}$

Note that the quadratic dependence of the absorption process to the average laser intensity was checked at different characteristic wavelengths (denoted as "power scan" in Figure 2 and Figure S3, and including 532 nm, Figure S4) to ascertain the two-photon nature of the monitored process at these wavelengths. ${ }^{76}$

EPR spin trapping (EPR-ST) studies. To further demonstrate the applicability of our newly designed chromophores for two-photon microfabrication, photoinduced radical generation upon continuous irradiation of dichloromethane solution of V-Shape was studied by means of EPR-ST spectroscopy. To facilitate identification of the generated radical, EPR signals and spectral simulations were systematically compared to those obtained with literature benchmarks. In addition to our aforementioned Norrish I (OXE2) and Norrish II (BDEBP) PIs, azobisisobutyronitrile (AIBN) was also used to verify the reliability of the approach. AIBN is known to efficiently produce a single radical species upon thermal or photodegradation, and should in principle lead to a unique EPR signal. Moreover, in order to facilitate radical detection, radical scavengers $\mathrm{N}$-tert-butyl- $\alpha$-phenylnitrone (PBN), and 5,5-dimethyl-1-pyrroline $\mathrm{N}$-oxide (DMPO) were used for each compound, and the results obtained with those two scavengers were systematically compared.

EPR spin trapping (EPR-ST) studies. To further demonstrate the applicability of our newly designed chromophores for two-photon microfabrication, photoinduced radical generation upon continuous irradiation of dichloromethane solution of V-Shape was studied by means of EPR-ST spectroscopy.
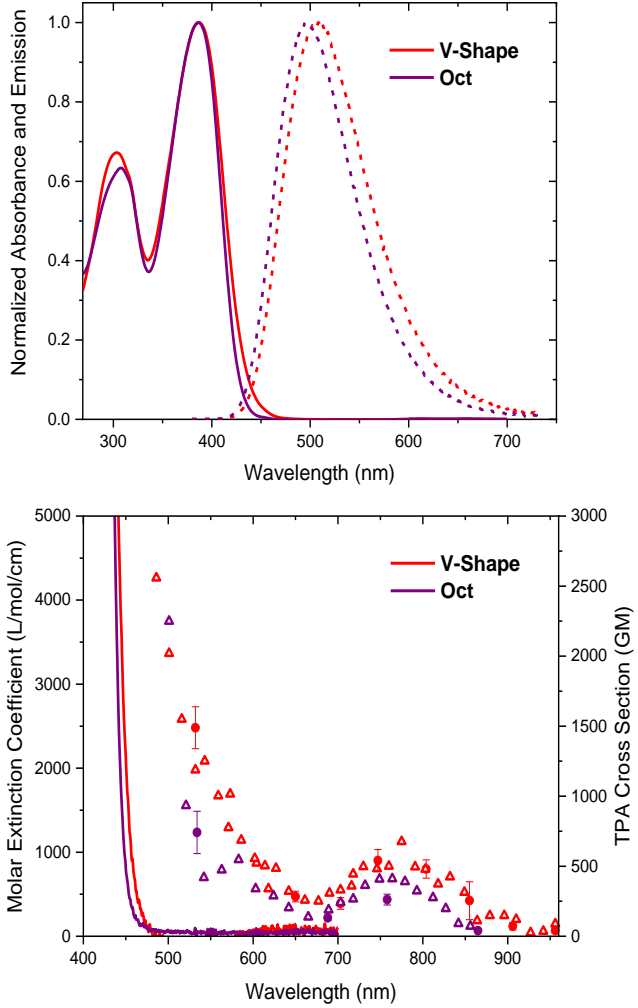

Figure 2. Normalized absorption (solid lines) and emission spectra (dashed lines) of V-Shape (red) and Oct (purple) in dichloromethane (top figure). The red-tail of OPA spectra (solid lines) along with TPA spectra of V-Shape (red) and Oct (purple) in dichloromethane obtained by wavelength (WL)scan measurement (empty triangles) at a constant incident power and power scan measurement (full circle) performed with four irradiation powers at each wavelength, by which it proves that the observed signal originates from TPA for the selected wavelengths (bottom figure).

To facilitate identification of the generated radical, EPR signals and spectral simulations were systematically compared to those obtained with literature benchmarks. In addition to our aforementioned Norrish I (OXE2) and Norrish II (BDEBP) PIs, azobisisobutyronitrile (AIBN) was also used to verify the reliability of the approach. AIBN is known to efficiently produce a single radical species upon thermal or photodegradation, and should in principle lead to a unique EPR signal. Moreover, in order to facilitate radical detection, radical scavengers $\mathrm{N}$-tert-butyl-a-phenylnitrone (PBN), and 5,5-dimethyl-1-pyrroline $\mathrm{N}$-oxide (DMPO) were used for each compound, and the results obtained with those two scavengers were systematically compared. 
As expected, a single radical species was obtained in the presence of AIBN, whatever scavenger considered. The features of the trapped radical species as monitored in either PBN $\left(a_{N}=14.1, a_{H}=2 G\right)$ or DMPO $\left(a_{N}=13.0 G\right.$, $a_{H}$ $=8.9 \mathrm{G}, \mathrm{a}_{\mathrm{H}}=1.5 \mathrm{G}$ ) are consistent with a radical species originating from the classical decomposition mechanism of AIBN, after possible oxidation with traces of oxygen contained within the medium as found in previous works with this initiator (see Figure S5). ${ }^{77}$

Irradiation of OXE2 gives a more complex pattern; in addition to the previously reported spin-adduct corresponding to an acetoxyl $\mathrm{COO}^{*}$ radical (adduct of PBN: $\mathrm{a}_{\mathrm{N}}=13.5$ $\mathrm{G}$ and $\mathrm{a}_{\mathrm{H}}=1.6 \mathrm{G}$; adduct of DMPO: $\mathrm{a}_{\mathrm{N}}=12.8 \mathrm{G}, \mathrm{a}_{\mathrm{H}}=10.3$ $\mathrm{G}$ and $\left.\mathrm{a}_{\mathrm{H}}=1.1 \mathrm{G}\right),{ }^{36,78}$ three other species were also identified. In the first, hyperfine coupling constants are consistent with the methyl radical $\mathrm{CH}_{3}{ }^{\bullet}$ (adduct of PBN: $\mathrm{a}_{\mathrm{N}}=$ $15.1 \mathrm{G}$ and $\mathrm{a}_{\mathrm{H}}=3.4 \mathrm{G}$; adduct of DMPO: $\mathrm{a}_{\mathrm{N}}=14.7 \mathrm{G}$ and $a_{H}=20.5 \mathrm{G}$ ), which agrees with a mechanism consisting in a $\mathrm{CO}_{2}$ elimination from the acetoxyl radical. A last adduct, which we attribute to iminyl radical resulting from the $\mathrm{N}-\mathrm{O}$ band photocleavage was similarly observed with both trapping agents (adduct of PBN: $\mathrm{aN}=13.9 \mathrm{G}$ and $\mathrm{aH}_{\mathrm{H}}$ $=2.3 \mathrm{G}$; adduct of DMPO: $\mathrm{aN}_{\mathrm{N}}=14.5 \mathrm{G}$ and $\mathrm{aH}_{\mathrm{H}}=15 \mathrm{G}$ ). In addition, an adduct of DMPO highlights the presence of another species consistent with reported values for acetyl radical $\left(\mathrm{a}_{\mathrm{N}}=13.0 \mathrm{G}, \mathrm{a}_{\mathrm{H}}=7.0 \mathrm{G}\right.$ and $\left.\mathrm{a}_{\mathrm{H}}=1.5 \mathrm{G}\right)$ even though the presence of this species is not fully understood. Note that this radical may also be present in PBN, but its expected feature may render it undistinguishable from the iminyl radical. In addition to these signals, a triplet signal due to the degradation of DMPO $\left(\mathrm{a}_{\mathrm{N}}=13.9 \mathrm{G}\right)^{79}$ is visible in the corresponding EPR spectrum (see Figure S5).

In contrast, strong similarities are seen for the dominant radical species obtained with BDEBP and V-Shape (see below and Figure 3). By analogy with previous studies and general agreement on Michler's ketone (or related molecules $^{80}$ ) photoinitiation mechanism, ${ }^{81}$ and taking in consideration the structural analogies between the former and V-Shape, we attribute the observed first radical species trapped with PBN to a C-centered radical in alpha position of the aliphatic chain, relative to the aniline group (aN-BDEBP $=14 \mathrm{G}$, a $_{\text {H-BDEBP }}=2.2 \mathrm{G}$; $\mathrm{a}_{\mathrm{N} \text {-v-Shape }}=14.6 \mathrm{G}$, a $_{\mathrm{H} \text {-v } \text {-Shape }}=$ $2.5 \mathrm{G}$ ). The hyperfine coupling constants indeed fully match those previously described in literature. ${ }^{82-83} \mathrm{~A} \mathrm{sec}-$ ond signal common to both Pls $\left(\mathrm{a}_{\mathrm{N}}=8.1 \mathrm{G}\right)$ can be attributed to a degradation of PBN, ${ }^{79}$ finally a third species is observed with BDEBP exclusively (aN-BDEBP $=14.8 \mathrm{G}$, ан-вDEBP $=4.6 \mathrm{G}$ ): its signature corresponds to a C-centered radical, located on the carbonyl moieties, and is typically encountered with benzophenone derivatives. ${ }^{84-85}$ It is therefore absent from V-Shape signature, the latter being devoid of a carbonyl function.

By analogy with literature, the first signal observed in DMPO (aN-BDEBP $=14.5 \mathrm{G}$, a $_{\text {H-BDEBP }}=21 \mathrm{G}$; $\mathrm{a}_{\mathrm{N}-\mathrm{V} \text {-Shape }}=14.5$ $\mathrm{G}$, aH-v-shape $=21.5 \mathrm{G}$ ) may correspond to adducts of the same species described with a possible superimposition of the two C-centered signals in the case of BDEBP. Due to the better ability of DMPO to trap N and O-centered radicals, we attribute the second and major species (aNBDEBP $=13.5 \mathrm{G}, \mathrm{a}_{\text {H-BDEBP }}=11.7 \mathrm{G}$; $\mathrm{a}_{\mathrm{N}-\mathrm{V} \text {-Shape }}=13.4 \mathrm{G}$, $\mathrm{a}_{\mathrm{H}-\mathrm{V}}$ -
Shape $=11.4 \mathrm{G}$ ) to an $\mathrm{N}$-centered (i.e. on the aniline nitrogen) cation radical. Eventually, a signal corresponding to the degradation of DMPO was observed for both PIs ( $a_{N}$ $=13.9 \mathrm{G}){ }^{79}$ These species are consistent with a mechanism consisting an initial electron transfer step, which then evolves through hydrogen abstraction from another PI molecule or the solvent. It constitutes another indication that V-Shape (and Oct, by extension) most probably proceed through an initial homo-intermolecular electron transfer, and that the resulting radical possibly evolves by hydrogen abstraction, a point that also receives support from TD-DFT computational modeling (vide infra).
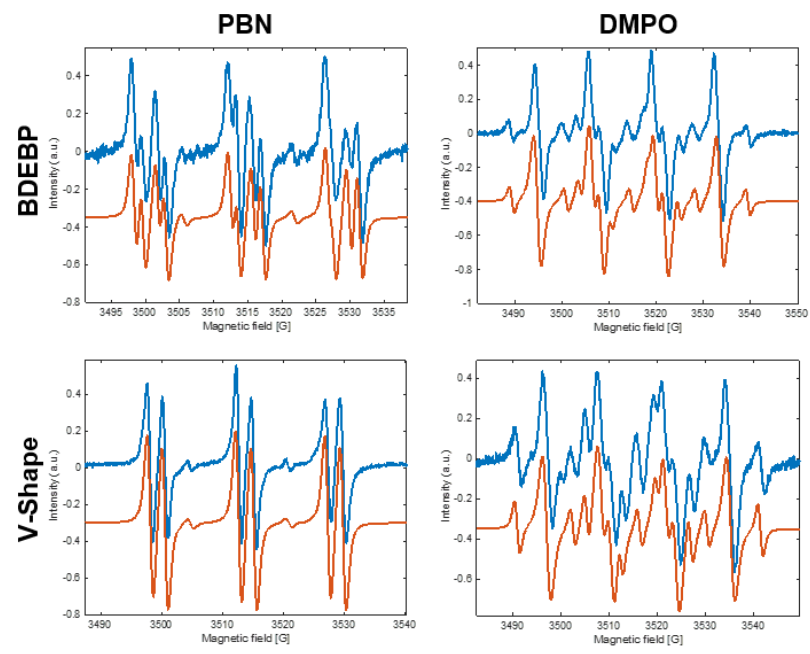

Figure 3. EPR spectra of spin trap adducts generated after irradiation of BDEBP and $\boldsymbol{V}$-Shape at $365 \mathrm{~nm}$ in presence of $P B N$ or DMPO in dichloromethane. Experimental spectra (blue) and simulated spectra (orange).

Electrochemical studies. The thermodynamic feasibility of such a photoredox process was further demonstrated by electrochemical methods. The oxidation and reduction potentials of V-Shape and Oct were determined by CV measurements carried out in deoxygenated dichloromethane in the presence of tetra- $n$-butylammonium $(0.1$ $\mathrm{mol} / \mathrm{L}$ ). As a general statement, the thermodynamic driving force for electron transfer (ET) can be calculated using the following equation, sometimes abusively referred to as the Rehm-Weller relation (see Equation 2): ${ }^{86}$

$\Delta G_{E T}=F\left[E^{\circ}\left(D^{+} / D\right)-E^{\circ}\left(A / A^{-}\right)-\Delta E_{00}\right]+w_{p}$

Where $F$ is the Faraday constant, $E^{\circ}\left(D^{+} / D\right)$ and $E^{\circ}(A /$ $A^{-}$) stand for the donor and acceptor oxidation and reduction potentials, respectively (or the lowest oxidation and reduction potential of the $\mathrm{PI}$, in the case of an homo-intermolecular process like here), $\Delta E_{00}(\mathrm{eV})$ is the so-called zero-zero transition energy, i.e. the energy difference between the vibrationally relaxed ground and excited states of the molecule, which corresponds in the case of a fluorophore to the crossing point of its normalized absorption and emission spectra, and $w_{p}$ is the Coulomb stabilization energy associated with the intermediate radical ion pair interaction energy with the solvent. The latter is usually neglected in a first approximation. 
As can be seen in Figure 4, the CV curves of V-Shape and Oct display similar signatures, with one irreversible oxidation wave observed at about $0.35 \mathrm{~V}$ and one irreversible reduction wave between -2.3 and $-2.5 \mathrm{~V}$ (see

Table 1). The irreversibility of these $\mathrm{CV}$ waves reveal the limited stability of both the oxidized and reduced species generated at the electrode interface. The cation radical moieties produced at $0.35 \mathrm{~V}$ on the $\mathrm{CV}$ curves of V-Shape and Oct was found to be unstable at all investigated scan rates $(100-20 \mathrm{~V} / \mathrm{s})$ and the existence of chemical reactions coupled to the electron transfer was revealed on the CV curves by the observation of additional waves on the return scan $(E<-0.5 \mathrm{~V})$. Both the oxidation potential and observed behavior agree well with the formation of an $(\mathrm{Hex})_{2} \mathrm{ArN}^{\circ+}$ cation radical. ${ }^{87}$ Density functional theory (DFT) calculations carried out on the oxidized and reduced forms of V-Shape suggest the formation of a stable radical anion, with an extensive delocalization of the electron density contributing to its stability whereas the radical cation is localized at the aniline moieties (see energetic diagram and spin densities given in Figure S6). The latter is prone to deprotonate spontaneously with a marked exergonicity at the aliphatic $\mathrm{C}$ adjacent to the aniline center, in good agreement with the conclusions drawn on the basis of EPR data. Calculated oxidation potentials agree well the ones measured experimentally (see ESI).

On the cathodic side, the reductions of V-Shape and Oct are observed at significantly different potential values. This shift of $\sim 150 \mathrm{mV}$ observed between those two irreversible waves is however fully consistent with the presence of an electron withdrawing bromine atom on VShape; which supports the conclusion that the electron transfer is centered in both cases on the conjugated polyaryl backbone.

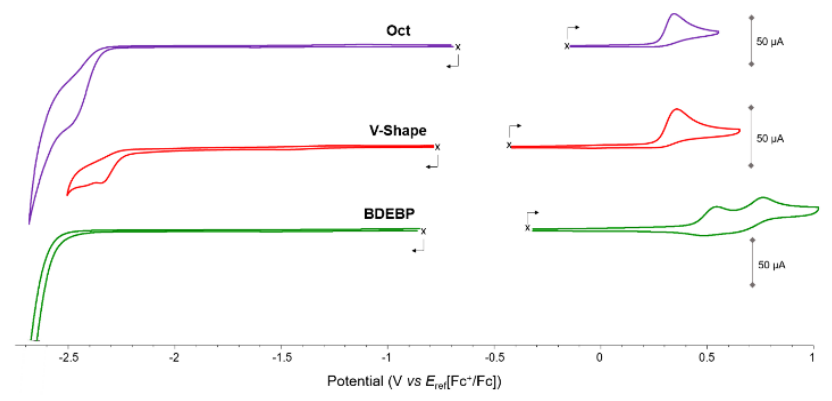

Figure 4. Cyclic voltammetry of V-Shape, Oct and BDEBP (1 $\mathrm{mmol} / \mathrm{L}$ ) in dichloromethane in the presence of tetra- $n$-butylammonium perchlorate $(0.1 \mathrm{~mol} / \mathrm{L}), E(V)$ vs. Eref $\left[F c / F c^{+}\right]$, at a vitreous carbon working electrode $\varnothing=3 \mathrm{~mm}, 298 \mathrm{~K}, \mathrm{v}=$ $0.1 \mathrm{~V} / \mathrm{s}$.

The distribution of spin densities obtained at the DFT/M06HF/6-31G(d,p) shown in Figure S6 indicates a stronger delocalization of the radical anion, hence more stable. Here again, the irreversibility of both $\mathrm{CV}$ waves reveals the unstable character of the electrogenerated an- ion radicals which could potentially be involved in hydrogen abstraction, radical-radical coupling reactions or in the hydrogenolysis of the carbon-halogen bond. ${ }^{88}$

From the spectroscopic data, $\Delta E_{00}$ is found at $440 \mathrm{~nm}$ and $433 \mathrm{~nm}$ for V-Shape and Oct respectively (2.82 and 2.87 $\mathrm{eV})$. From Equation 2, a standard Gibbs energy for the ET between two V-Shape or Oct molecules can be calculated, corresponding to -11.4 and $-2.3 \mathrm{~kJ} / \mathrm{mol}$. Similar experiments were also performed on BDEBP (see Figure 4), unfortunately reduction potentials lie outside of the accessible electrochemical window, precluding calculation of the free energy for the homo-intermolecular electron transfer in this case.

CV measurements were also conducted on the monomer. No accessible oxidation or reduction potential could be recorded within the electrochemical window (see Figure S7), a priori discarding the possibility of a photoinduced ET with any of the PIs during the photoinitiation process, in contrast with other literature reports. ${ }^{89}$ This was further confirmed by a Stern-Volmer experiment ${ }^{90}$ where evolution of the luminescence intensity of V-Shape and Oct was monitored upon incremental addition of increasing monomer (DPPHA) concentration (see Figure S8). As no evolution was seen even at high monomer concentration, it can be concluded that no electron or energy transfer takes place between the singlet excited state of V-Shape or Oct and the monomer.

In summary, these data clearly outline the possibility of an exergonic photoinduced disproportionation for V-Shape and Oct. These data, in line with EPR results, strongly suggest that this electron transfer constitutes the initial step leading to radical formation and polymerization initiation in the microfabrication process.

Photolysis experiments. Spectroscopic study of the photolysis of Pls was then undertaken in order to gain more insight into the kinetics of the radical generation process, and to test how the latter is influenced by the presence of monomers. It has been shown in previous literature reports that, in these strictly anaerobic conditions, photodegradation of Pls proceeds through formation of photoinduced radical species which evolve, in the absence of scavenging species, to the build-up of photolysis products. Thus, photobleaching rate can be correlated to radical photogeneration efficiency, as recently exemplified by Lalevée et al. or Liska et al. ${ }^{91-95}$ Kinetics of chromophores photobleaching upon continuous irradiation in the ICT band were investigated upon irradiation with a LED at $365 \mathrm{~nm}$, by measuring the in-time decay of luminescence signal intensity, using thoroughly deaerated (five freeze-pump-thaw cycles with a secondary vacuum pump) solution of V-Shape and Oct in dichloromethane. Evolution of the UV-vis spectra were also monitored in parallel (see Figure 5 and Figure S9).

In the present case, large changes of the isosbestic point at around $360 \mathrm{~nm}$ in the absorption spectrum throughout the experiment could be evidenced for both V-Shape and Oct, with a similar evolution profile in both cases. This indicates a non-straightforward degradation process involving multiple competitive pathways. Two steps at least can be distinguished: in the first 10 minutes, a decrease of ICT band, which can be attributed to the decomposition of $\mathbf{V}$ - 
Shape (or Oct), is associated to the concomitant increase of the absorbance at $359 \mathrm{~nm}$ and $325 \mathrm{~nm}$. Then, while decrease of the ICT band slowly proceeds forward, the newly formed bands at $359 \mathrm{~nm}$ and $325 \mathrm{~nm}$ in turn start to decrease, leading to a drift of the isosbestic point. This double evolution suggests the formation (first step) and consumption (second step) of degradation products of the Pls.

In spite of the relatively intricate profile of photo-induced degradation process, attempts were made to fit the first ten minutes of the recorded evolutions to a simple kinetic model. A relatively good correlation was obtained in the case of V-Shape and Oct with an apparent first order reaction (see Figure 6 and Figure S10), ${ }^{96}$ which seemingly disagrees with the hypothesis of bimolecular electron transfer. However, one has to take into account that the range of studied concentrations (ca. $10^{-6} \mathrm{M}$ ), as imposed by the spectrofluorimetric experiment, may affect the radical generation mechanism: in particular, such low concentrations are reportedly known to strongly disfavor bimolecular mechanisms in favor of direct hydrogen abstraction with the solvent, which might account for the observed discrepancies. ${ }^{97}$

Photolytic rates were found to follow the trend: BDEBP >> Oct $>$ V-Shape. This indicates that BDEBP generates radicals more efficiently than Oct which itself is slightly more efficient than V-Shape. This result is consistent with the fact that the molecular design of V-Shape and Oct was primarily aimed at improving $\sigma_{T P A}$ rather than the radical generation efficiency. The difference between VShape and Oct might be attributed to the extra arm of Oct since the radicals are expected to be formed in a position of the aniline.
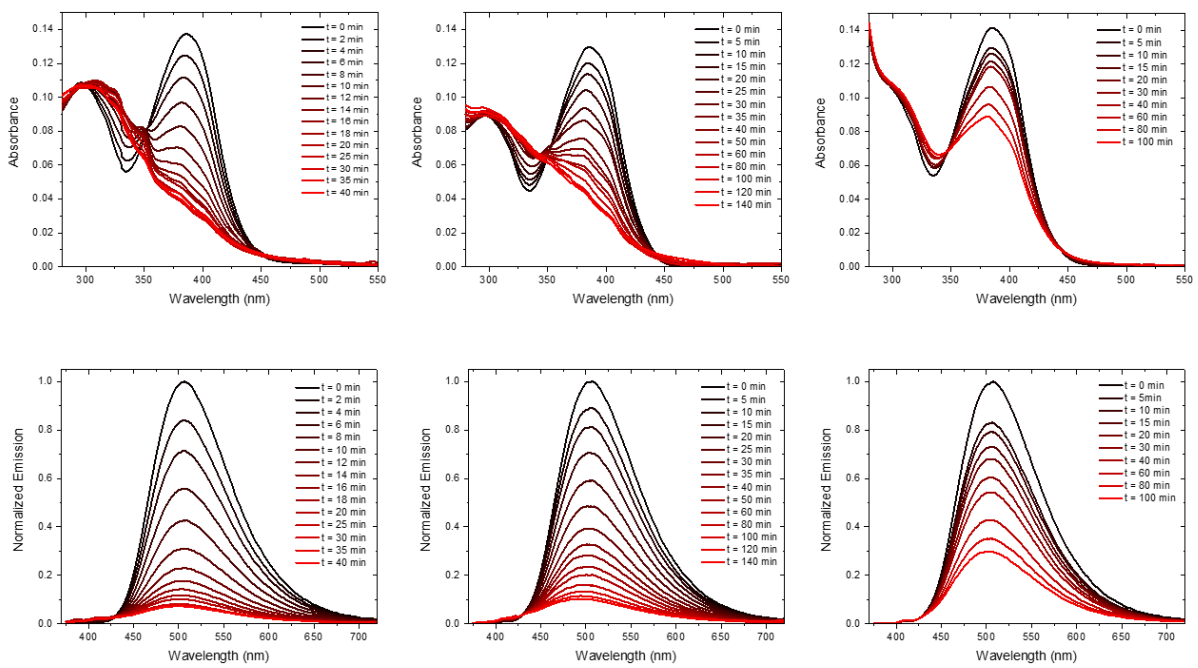

Figure 5. Photolysis studies (UV-Vis and fluorescence) of V-Shape in degassed dichloromethane under $365 \mathrm{~nm}$ irradiation, 40 $\mathrm{mW}$ in absence or presence of Ethyl Acrylate (EA). [V-Shape] $=1.4 \times 10^{-6} \mathrm{~mol} / \mathrm{L} ;[E A]=0 \mathrm{~mol} / \mathrm{L}$ (left); $1.4 \times 10^{-3} \mathrm{~mol} / \mathrm{L}$ (center) and $7 \times 10^{-3} \mathrm{~mol} / \mathrm{L}$ (right).

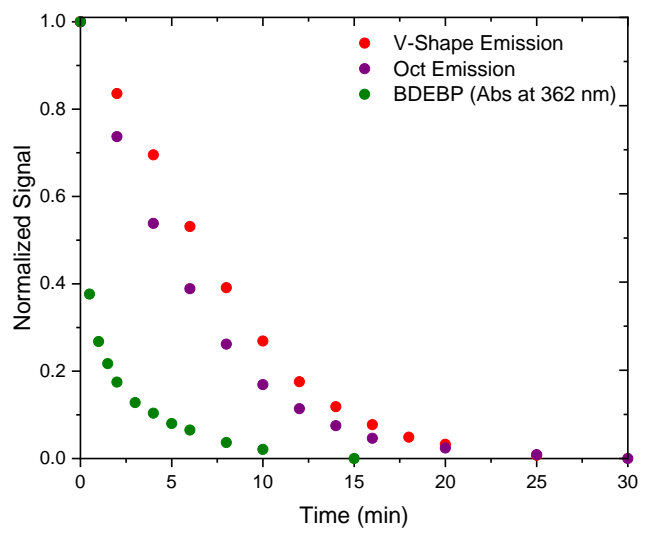

Figure 6. Photolysis of V-Shape (red), Oct (purple) and BDEBP (green) in degassed dichloromethane under $365 \mathrm{~nm}$ irradiation, $40 \mathrm{~mW}$ at isoabsorbance concentration at 365 nm. $[$ V-Shape $]=1.4 \times 10^{-6} \mathrm{~mol} / \mathrm{L} ;[$ Oct $]=8.6 \times 10^{-7} \mathrm{~mol} / \mathrm{L}$ and $[B D E B P]=2.6 \times 10^{-6} \mathrm{~mol} / \mathrm{L}$.

Moreover, it was unambiguously shown that addition of monomer (Ethyl Acrylate EA and DPPHA) markedly slows down the photobleaching of V-Shape and Oct (see Figure 7 and Figure S11). The extent of this slow-down appears to be monomer concentration dependent. Taken together with the electrochemical and Stern-Volmer data (see above), we hypothesize from this last result that the photoinitiation mechanism takes place in two consecutive steps $\mathrm{i} /$ an initial photoinduced disproportionation of PI, generating the corresponding radical anion and radical cation pairs; in the absence of monomer or other scavenging species, these species evolve towards terminal degradation products, ii/ an electron transfer towards (or alternatively, radical addition unto) monomer molecules, which restores at least a part of the chromophore (one or two arms) to its initial state and prevents its evolution towards degradation products so that the initial signal of fluorescence and absorbance is at least partially restored. 


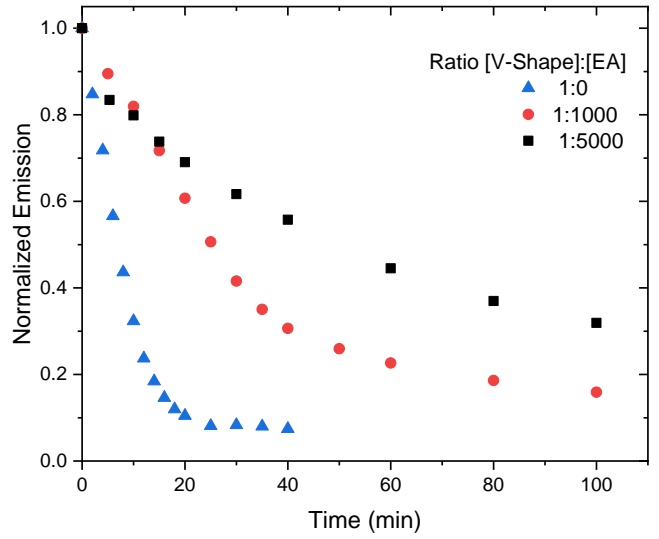

Figure 7. Photolysis kinetics followed by fluorescence measurement of V-Shape (top) and Oct (bottom) in degassed dichloromethane under $365 \mathrm{~nm}$ irradiation, $40 \mathrm{~mW}$ in absence or presence of EA. [V-Shape] $=1.4 \times 10^{-6} \mathrm{~mol} / \mathrm{L} ;[\mathrm{Oct}]=8.6$ $\times 10^{-7} \mathrm{~mol} / \mathrm{L} ;[E A]=0 \mathrm{~mol} / \mathrm{L}$ (blue); $1.4 \times 10^{-3} \mathrm{~mol} / \mathrm{L}$ (red); $7 \times$ $10^{-3} \mathrm{~mol} / \mathrm{L}$ (black).

Two-photon induced 3D microfabrication. With this information in hands, we finally studied the potential of VShape and Oct for the two-photon polymerization (TPP) of acrylic monomers. Our main concern was to compare the polymerization threshold $\left(P_{\text {th }}\right)$ obtained with V-Shape and Oct with benchmark Pls, and see whether lowering of this value could be obtained without affecting the accessible resolution.

As presented earlier, design of the newly synthesized PIs involves hexyl substitution on the terminal aniline moieties. The purpose of this long alkyl group was to improve chromophore solubility in the photoresist formulation, such long $\pi$-conjugated structures featuring large $\sigma_{T P A}$ being particularly prone to aggregation because of $\pi$-stacking. To take advantage of this feature and maximize PI solubility, a reactive "diluent" was introduced within the resist formulation, in the form of 1,10-decanediol diacrylate (DDA), a difunctionnal acrylic monomer featuring a long fatty alkyl chain. The rest of the resist (80wt\%) consisted in dipentaerythritol penta-/hexa-acrylate (DPPHA). The latter was chosen as its high functionality provides an excellent crosslinking density, which improves photoresist sensitivity as well as mechanical strength and reduces shrinkage of the generated pattern. ${ }^{98}$

This formulation afforded a maximal $1 \mathrm{wt} \%(10.9 \mathrm{mmol} / \mathrm{g})$ solubility of V-Shape upon direct mixing with the monomers. In contrast, the solubility of Oct remained limited to $0.66 \mathrm{wt} \%(5.4 \mathrm{mmol} / \mathrm{g})$ and was not improved with addition of co-solvent and sonication which we attribute to a larger $\pi$-conjugated backbone.

In order to evaluate $P_{\text {th }}$ and minimal accessible size of photogenerated polymers, two types of objects were fabricated. We considered that polymerization threshold was reached when the photogenerated structures could uphold the monomer washing step without undergoing significant distortion (see Figure 8d). As a first model structure, $20 \mu \mathrm{m}$ lines were printed directly at the surface of the coverslip paying close attention to the level of the laser focus position in order to be exactly at or slightly above the substrate/photoresist interface and thus measuring the correct linewidth while maintaining a good adhesion to the substrate. Figure $8 d$ is a SEM image of periodic lines fabricated with decreasing power at $40 \mu \mathrm{m} / \mathrm{s}$ writing speed. Although suspended lines are often preferred in literature for related studies, ${ }^{99-100}$ this choice of structures present the advantage of being extremely rapid to implement, on the one hand, and to reduce the impact of the shrinkage on the measurement of the linewidth on the other (structures being stabilized by anchoring to the coverslip). ${ }^{101}$ Moreover, it limits the potential deformation due to spherical aberrations. ${ }^{15}$

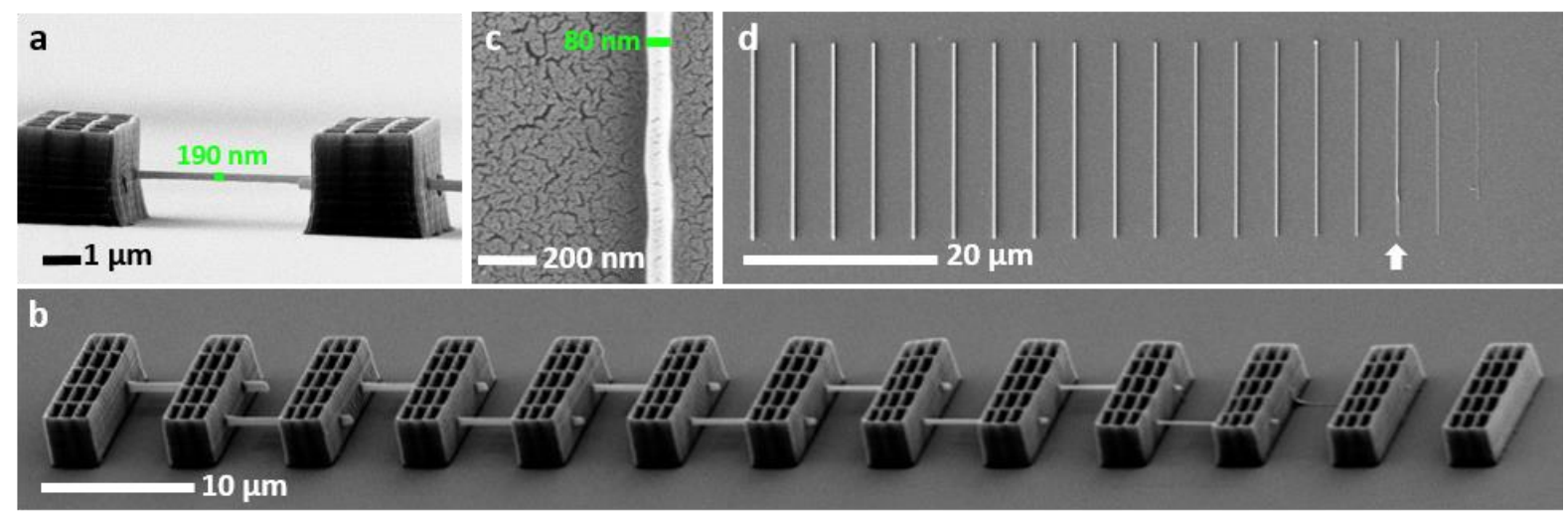

Figure 8. SEM pictures of representative structures generated by TPP for the measurements of the polymerization thresholds, the linewidths and the line heights. Scan speed: $40 \mu \mathrm{m} / \mathrm{s}$. (a) Grazing view of a suspended line, photoresist: DPPHA:DDA:VShape (79.2:19.8:1), $P_{a v} 103 \mu \mathrm{W}$ (b) $45^{\circ}$ view of suspended lines, photoresist: DPPHA:DDA:V-Shape (79.2:19.8:1), Pav from 257 to $82 \mu W$ (c) Top view of a line written at the surface of the coverslip, photoresist: DPPHA:DDA:Oct (79.5:19.8:0.7), Pav 96 $\mu W(d)$ Top view of lines written at the surface of the coverslip, the white arrow points to the line at the threshold, photoresist: DPPHA:DDA:Oct (79.5:19.8:0.7), Pav from 303 to $85 \mu \mathrm{W}$. 
However, it does not provide reliable measurement of the line heights. Thus for comparison, suspended lines were also printed to check for compliance with the results obtained with attached lines, and enable estimation of the aforementioned parameter. $13 \mu \mathrm{m}$ lines were hanged between $5 \mu \mathrm{m}$-separated blocks at $1.5 \mu \mathrm{m}$ from the surface (see Figure $8 a$ and $b$ ) to ensure good mechanical stability. The general protocol used for systematic measurement of line width and height are reported in the experimental section and detailed in ESI (see Figure S12). Basically, a Python script was designed to provide statistical treatment on a large number of measurements. Generally speaking, no significant difference was found between suspended and surface grafted lines for what concerns linewidth, while the line height and aspect ratio could be measured on suspended lines exclusively, with a value of ca. 2.7 , typical of TPP using high NA objective. ${ }^{102}$ (see Figure S13). However, it could be noted that the width of the suspended lines is slightly inferior to the width of the equivalent lines attached to the coverslip at low laser power (near threshold). This could be due to moderate shrinkage of the structure, more visible at low monomer conversion. In the following, we thus chose to focus on surface grafted lines for the determination of $P_{\text {th }}$.

Table 2. Comparison of the obtained $P_{\text {th }}$ between the studied photoresists. Composition: DPPHA:DDA (80:20) with 5.4 $\mu \mathrm{mol}$ Pl/g; scanning speed: $40 \mu \mathrm{m} / \mathrm{s}$.

\begin{tabular}{ll}
\hline PI & TPP $P_{\text {th }}(\mu \mathrm{W})$ \\
\hline V-Shape & 120 \\
Oct & 90 \\
BDEBP & 550 \\
ITX & 690 \\
OXE2 & 480 \\
\hline
\end{tabular}

Following this procedure we compared $\mathrm{P}_{\text {th }}$ between $\mathrm{V}$ Shape, Oct and our three benchmark compounds, in the monomer mixture described above (see Table 2).

It clearly appears that the polymerization thresholds are significantly improved when comparing Oct and V-Shape to the benchmarks. Threshold is lowered by approximately a factor 5-6 as compared to BDEBP, 6-8 as compared to ITX and 4-5 as compared to OXE2 for V-Shape and Oct respectively. This improvement could be explained by the much increased $\sigma$ TPA of the newly designed PIs as compared to commercially available benchmarks. It clearly indicates that $\mathbf{V}$-Shape and Oct constitute a very promising basis for the future development of improved PIs for two-photon induced polymerization.

In spite of a $\sigma_{T P A}$ more than one order of magnitude higher for V-shape and Oct than for BDEBP, $P_{\text {th }}$ is only improved by a factor of ca. 5. The lower absorption of BDEBP seems partly compensated by its better radical generation ability (vide supra). A similar reason might also account for the lower threshold of Oct as compared with V-Shape, while $\sigma_{T P A}$ measurements would suggest an opposite trend.
In a last set of experiments, dependence of the accessible structure size to irradiance was evaluated for each compound.

As expected, the linewidth increases upon increasing the laser power used during the microfabrication process. Although the observed evolution looks at first sight to be very dependent on the nature of the $\mathrm{PI}$, the impression vanishes when the evolution is plotted not versus the absolute laser beam intensity, but versus the ratio of applied power in regard with the $P_{\text {th }}$ value (see inset in Figure 9 and Figure S14). Using this representation, it becomes apparent that the photoresists display a power-dependence pattern that is similar for all PIs: in all cases, initial evolution of the structure dimension can be modeled using the "beam shape dependence function" presented below (see Equation 3) for power that does not exceed twice $P_{\text {th. }}{ }^{30,103}$

$v_{i}=w_{x y} \sqrt{2 \ln \frac{P_{i}}{P_{t h}}}(3)$

Where $v_{i}$ is the voxel width obtained with the laser power $P_{i}, w_{x y}$ is the beam waist in the $\mathrm{x}-\mathrm{y}$ plane, and $P_{t h}$ is the TPP threshold. As shown in Figures S15 and 16, the obtained linewidths are satisfactorily reproduced with Equation 3.

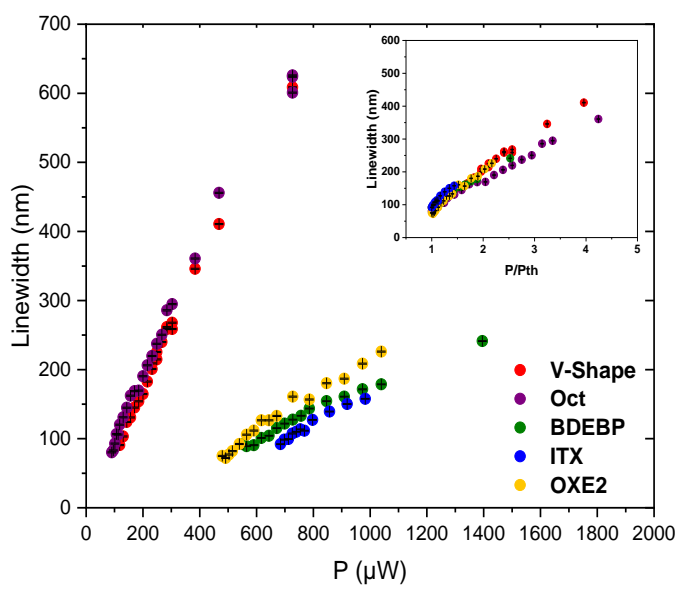

Figure 9. Linewidth versus average laser power and ratio average power/threshold power (inset) for the five studied photoresists.

Finally, in order to illustrate the potential of the newly synthesized photoresists for low-threshold two-photon printing of sub-100 nm resolution structures, we envisioned the fabrication of a $250 \mathrm{~nm}$ periodic grating and cubic woodpiles as those presented in Figure 10. A lateral resolution as small as $80 \mathrm{~nm}$ and an axial resolution as small as $190 \mathrm{~nm}$ were thus demonstrated using a low-cost subnanosecond pulsed $532 \mathrm{~nm}$ laser (see Figure 8a, c and Figure 10), proving that excellent resolution is maintained with our system. 

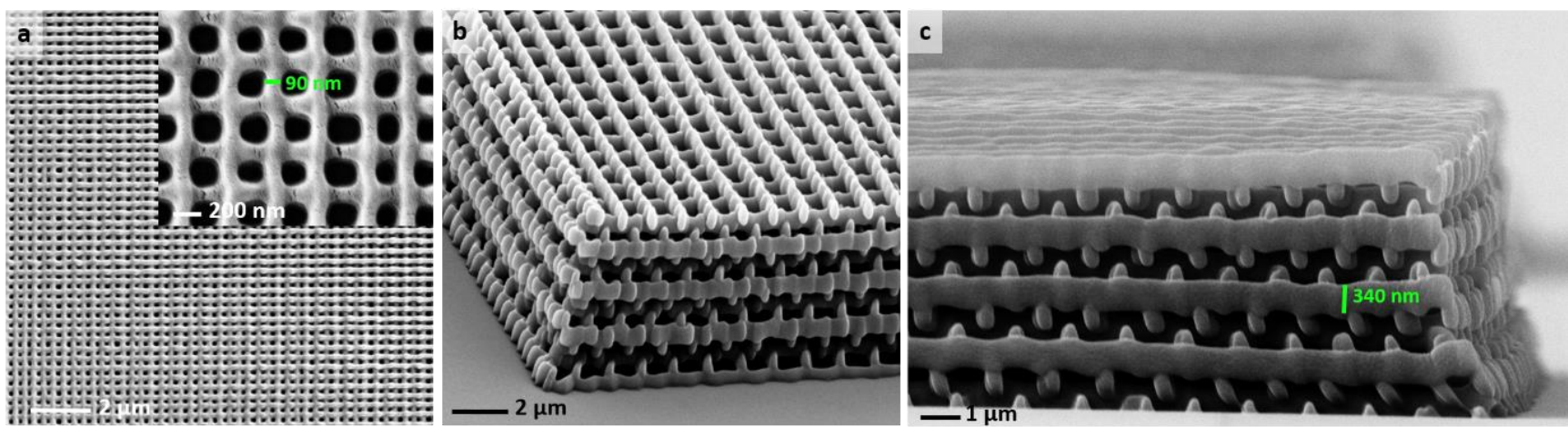

Figure 10. SEM pictures of representative structures generated by TPP. Writing speed $40 \mu \mathrm{m} / \mathrm{s}$, photoresist: DPPHA:DDA:VShape (79.2:19.8:1) (a) Top view of a $250 \mathrm{~nm}$ periodic grating with a close-up in the upper right corner, $P_{a v} 103 \mu \mathrm{W}$ (b) $45^{\circ}$ view of a $700 \mathrm{~nm}$-cubic woodpile, $P_{a v} 125 \mu \mathrm{W}$ (c) Grazing view of a $700 \mathrm{~nm}$-cubic woodpile, $P_{a v} 125 \mu \mathrm{W}$

\section{CONCLUSIONS}

In conclusion, we show that highly efficient radical twophoton initiators can be obtained taking advantage of near-resonant enhancement of the two-photon cross-section efficiency. Taking a simple m-conjugated structure,

that displays intense one-photon absorption in the blue part of the UV-vis spectrum, yet only moderate two-photon efficiency in the regular TPA range (with $\lambda_{\text {TPA }}=2 \times \lambda_{\text {OPA }}$, ca. $800 \mathrm{~nm}$ ), we show that it is possible to obtain $\sigma$ TPA values that reach, for one of the studied compounds, 1500 GM at the $532 \mathrm{~nm}$ operating wavelength of the laser (close to NOPA) used in our microfabrication setup.

Owing to their extended $\pi$-conjugated structures, the as designed Pls have accessible electrochemical potential in oxidation as well as in reduction, rendering photoinduced disproportionation thermodynamically allowed. As a result, photoinduced radical generation takes place upon $\mathrm{PI}$ irradiation, which can be used to efficiently initiate polymerization reactions. Both PIs being functionalized by long alkyl chain that favors their solubility in organic media, they can be readily solubilized within acrylic mixtures for direct use in two-photon microfabrication without additional preparation step. We illustrate the benefit of this approach by comparing the polymerization threshold obtained with both newly synthesized PIs to Norrish I and Norrish II type ones classically used for two-photon microfabrication with sub-nanosecond pulsed $532 \mathrm{~nm}$ lasers. Using similar photoresist formulation, the polymerization threshold was lowered up to eight times with the reported new PIs as compared to benchmarks, similar to some of the best reported Pls to date, in spite of a comparatively lower radical generation efficiency. Finally, we show that the improvement in polymerization threshold is not obtained at the expense of resolution, as illustrated with thefabrication of thin bridges and periodic structures with periodic distance of $250 \mathrm{~nm}$ which makes both new PIs (V-
Shape and Oct) excellent candidates for massive parallel printing of large surfaces.

\section{Funding Sources}

C.A. thanks the Agence Nationale de la Recherche ANR for financial support (PhD grant) in the framework of the "New3DPrint" project (ANR-17-CE08-0037). This work has received funding from the European Union's Horizon 2020 research and innovation program under grant agreement No 780278. The dissemination of results herein reflects only the author's view and the European Commission is not responsible for any use that may be made of the information it contains.

\section{Notes \\ The authors declare no competing financial interest. Patent Pending, Registry number FR2007303}

\section{ACKNOWLEDGMENT}

The authors thank the Centre Régional de Mesures Physiques de l'Ouest for the high resolution mass spectrometry measurements and the Pôle Scientifique de Modélisation Numérique/Institut Universitaire de France (PSMN/IUF) for computational resources.

\section{REFERENCES}

1. Tofail, S. A. M.; Koumoulos, E. P.; Bandyopadhyay, A.; Bose, S.; O’Donoghue, L.; Charitidis, C., Additive manufacturing: scientific and technological challenges, market uptake and opportunities. Materials Today 2018, 21 (1), 22-37.

2. Vaidya, S.; Ambad, P.; Bhosle, S., Industry 4.0 - A Glimpse. Procedia Manufacturing 2018, 20, 233-238.

3. Ligon, S. C.; Liska, R.; Stampfl, J.; Gurr, M.; Mülhaupt, R., Polymers for 3D Printing and Customized Additive Manufacturing. Chemical Reviews 2017, 117 (15), 1021210290.

4. Melchels, F. P. W.; Domingos, M. A. N.; Klein, T. J.; Malda, J.; Bartolo, P. J.; Hutmacher, D. W., Additive manufacturing of tissues and organs. Progress in Polymer Science 2012, 37 (8), 1079-1104.

5. Narayan, R. J.; Doraiswamy, A.; Chrisey, D. B.; Chichkov, B. N., Medical prototyping using two photon polymerization. Materials Today 2010, 13 (12), 42-48. 
6. Bos, F.; Wolfs, R.; Ahmed, Z.; Salet, T., Additive manufacturing of concrete in construction: potentials and challenges of 3D concrete printing. Virtual and Physical Prototyping 2016, 11 (3), 209-225.

7. $\quad$ Mehrpouya, M.; Dehghanghadikolaei, A.; Fotovvati, B.; Vosooghnia, A.; Emamian, S. S.; Gisario, A., The Potential of Additive Manufacturing in the Smart Factory Industrial 4.0: A Review. Applied Sciences 2019, 9 (18), 3865.

8. Attaran, M., The rise of 3-D printing: The advantages of additive manufacturing over traditional manufacturing. Business Horizons 2017, 60 (5), 677-688.

9. Bikas, H.; Stavropoulos, P.; Chryssolouris, G., Additive manufacturing methods and modelling approaches: a critical review. The International Journal of Advanced Manufacturing Technology 2016, 83 (1), 389-405.

10. Chatham, C. A.; Long, T. E.; Williams, C. B., A review of the process physics and material screening methods for polymer powder bed fusion additive manufacturing. Progress in Polymer Science 2019, 93, 68-95.

11. Lim, M. P.; Guo, X.; Grunblatt, E. L.; Clifton, G. M.; Gonzalez, A. N.; LaFratta, C. N., Augmenting mask-based lithography with direct laser writing to increase resolution and speed. Opt. Express 2018, 26 (6), 7085-7090.

12. Stansbury, J. W.; Idacavage, M. J., 3D printing with polymers: Challenges among expanding options and opportunities. Dental Materials 2016, 32 (1), 54-64.

13. Maruo, S.; Ikuta, K., Submicron stereolithography for the production of freely movable mechanisms by using singlephoton polymerization. Sensors and Actuators A: Physical 2002, 100 (1), 70-76.

14. Park, S.-H.; Yang, D.-Y.; Lee, K.-S., Two-photon stereolithography for realizing ultraprecise three-dimensional nano/microdevices. Laser \& Photonics Reviews 2009, 3 (1-2), $1-11$.

15. Burmeister, F.; Steenhusen, S.; Houbertz, R.; Zeitner, U. D.; Nolte, S.; Tünnermann, A., Materials and technologies for fabrication of three-dimensional microstructures with sub$100 \mathrm{~nm}$ feature sizes by two-photon polymerization. Journal of Laser Applications 2012, 24 (4), 042014.

16. Pearre, B. W.; Michas, C.; Tsang, J.-M.; Gardner, T. J.; Otchy, T. M., Fast micron-scale 3D printing with a resonantscanning two-photon microscope. Additive Manufacturing 2019, 30, 100887.

17. Farsari, M.; Chichkov, B. N., Two-photon fabrication. Nature Photonics 2009, 3 (8), 450-452.

18. Poocza, L.; Gottschaldt, M.; Markweg, E.; Hauptmann, N.; Hildebrand, G.; Pretzel, D.; Hartlieb, M.; Reichardt, C.; Kübel, J.; Schubert, U. S.; Mollenhauer, O.; Dietzek, B.; Liefeith, K., Optimized Photoinitiator for Fast Two-Photon Absorption Polymerization of Polyester-Macromers for Tissue Engineering Advanced Engineering Materials 2017, 19 (3), 1600686.

19. Maruo, S.; Fourkas, J. T., Recent progress in multiphoton microfabrication. Laser \& Photonics Reviews 2008, 2 (1-2), 100-111.

20. Houbertz, R.; Fröhlich, L.; Popall, M.; Streppel, U.; Dannberg, P.; Bräuer, A.; Serbin, J.; Chichkov, B. N., InorganicOrganic Hybrid Polymers for Information Technology: from Planar Technology to 3D Nanostructures. Advanced Engineering Materials 2003, 5 (8), 551-555.

21. Stender, B.; Mantei, W.; Houbertz, R., From Lab to Fab - High-Precision 3D Printing. Laser Technik Journal 2017, 14 (2), 20-23.
22. Stender, B.; Hilbert, F.; Dupuis, Y.; Krupp, A.; Mantei, W.; Houbertz, R., Manufacturing strategies for scalable highprecision 3D printing of structures from the micro to the macro range. In Advanced Optical Technologies, 2019; Vol. 8, p 225.

23. Houbertz, R.; Steenhusen, S.; Stichel, T.; Sextl, G., TwoPhoton Polymerization of Inorganic-Organic Hybrid Polymers as Scalable Technology Using Ultra-Short Laser Pulses. In Coherence and Ultrashort Pulse Laser Emission Duarte, F. J., Ed. InTech: 2010.

24. Houbertz, R.; Satzinger, V.; Schmid, V.; Leeb, W.; Langer, G., Optoelectronic printed circuit board: 3D structures written by two-photon absorption. SPIE: 2008; Vol. 7053.

25. Xu, B.; Du, W.-Q.; Li, J.-W.; Hu, Y.-L.; Yang, L.; Zhang, C.C.; Li, G.-Q.; Lao, Z.-X.; Ni, J.-C.; Chu, J.-R.; Wu, D.; Liu, S.-L.; Sugioka, K., High efficiency integration of three-dimensional functional microdevices inside a microfluidic chip by using femtosecond laser multifoci parallel microfabrication. Scientific Reports 2016, 6 (1), 19989.

26. Geng, Q.; Wang, D.; Chen, P.; Chen, S.-C., Ultrafast multi-focus 3-D nano-fabrication based on two-photon polymerization. Nature Communications 2019, 10 (1), 2179.

27. Anseth, K. S.; Bowman, C. N.; Peppas, N. A., Polymerization kinetics and volume relaxation behavior of photopolymerized multifunctional monomers producing highly crosslinked networks. Journal of Polymer Science Part A: Polymer Chemistry 1994, 32 (1), 139-147.

28. Kuebler, S. M.; Rumi, M.; Watanabe, T.; Braun, K.; Cumpston, B. H.; Heikal, A. A.; Erskine, L. L.; Thayumanavan, S.; Barlow, S.; Marder, S. R.; Perry, J. W., Optimizing Two-Photon Initiators and Exposure Conditions for Three-Dimensional Lithographic Microfabrication. Journal of Photopolymer Science and Technology 2001, 14 (4), 657-668.

29. Ligon, S. C.; Husár, B.; Wutzel, H.; Holman, R.; Liska, R., Strategies to Reduce Oxygen Inhibition in Photoinduced Polymerization. Chemical Reviews 2014, 114 (1), 557-589.

30. Sun, H.-B.; Takada, K.; Kim, M.-S.; Lee, K.-S.; Kawata, S., Scaling laws of voxels in two-photon photopolymerization nanofabrication. Applied Physics Letters 2003, 83 (6), 11041106.

31. Waller, E. H.; Von Freymann, G., Spatio-Temporal Proximity Characteristics in 3D $\mu$-Printing via Multi-Photon Absorption. Polymers 2016, 8 (8), 297.

32. Schafer, K. J.; Hales, J. M.; Balu, M.; Belfield, K. D.; Van Stryland, E. W.; Hagan, D. J., Two-photon absorption crosssections of common photoinitiators. Journal of Photochemistry and Photobiology A: Chemistry 2004, 162 (2), 497-502.

33. Cumpston, B. H.; Ananthavel, S. P.; Barlow, S.; Dyer, D. L.; Ehrlich, J. E.; Erskine, L. L.; Heikal, A. A.; Kuebler, S. M.; Lee, I. Y. S.; McCord-Maughon, D.; Qin, J.; Röckel, H.; Rumi, M.; Wu, X.L.; Marder, S. R.; Perry, J. W., Two-photon polymerization initiators for three-dimensional optical data storage and microfabrication. Nature 1999, 398 (6722), 51-54.

34. Fouassier, J.-P.; Morlet-Savary, F.; Lalevée, J.; Allonas, $\mathrm{X}$; Ley, C., Dyes as Photoinitiators or Photosensitizers of Polymerization Reactions. Materials 2010, 3 (12), 5130-5142. 35. Lee, K.-S.; Kim, R. H.; Yang, D.-Y.; Park, S. H., Advances in 3D nano/microfabrication using two-photon initiated polymerization. Progress in Polymer Science 2008, 33 (6), 631681.

36. Zhou, R.; Malval, J.-P.; Jin, M.; Spangenberg, A.; Pan, H.; Wan, D.; Morlet-Savary, F.; Knopf, S., A two-photon active chevron-shaped type I photoinitiator designed for 3D stereolithography. Chemical Communications 2019, 55 (44), 6233-6236. 
37. Mendonca, C. R.; Correa, D. S.; Baldacchini, T.; Tayalia, P.; Mazur, E., Two-photon absorption spectrum of the photoinitiator Lucirin TPO-L. Applied Physics A: Materials Science Processing \$V 90 2007, (4), 633-636.

38. $\quad$ Qiu, W.; Hu, P.; Zhu, J.; Liu, R.; Li, Z.; Hu, Z.; Chen, Q.; Dietliker, K.; Liska, R., Cleavable Unimolecular Photoinitiators Based on Oxime-Ester Chemistry for Two-Photon ThreeDimensional Printing. ChemPhotoChem 2019, 3 (11), 10901094.

39. Tehfe, M.-A.; Dumur, F.; Graff, B.; Morlet-Savary, F.; Fouassier, J.-P.; Gigmes, D.; Lalevée, J., New Push-Pull Dyes Derived from Michler's Ketone For Polymerization Reactions Upon Visible Lights. Macromolecules 2013, 46 (10), 37613770 .

40. Fast, D. E.; Lauer, A.; Menzel, J. P.; Kelterer, A.-M.; Gescheidt, G.; Barner-Kowollik, C., Wavelength-Dependent Photochemistry of Oxime Ester Photoinitiators. Macromolecules 2017, 50 (5), 1815-1823.

41. Ummethala, G.; Jaiswal, A.; Chaudhary, R. P.; Hawal, S.; Saxena, S.; Shukla, S., Localized polymerization using single photon photoinitiators in two-photon process for fabricating subwavelength structures. Polymer 2017, 117, 364-369.

42. Belfield, K. D.; Schafer, K. J.; Liu, Y.; Liu, J.; Ren, X.; Stryland, E. W. V., Multiphoton-absorbing organic materials for microfabrication, emerging optical applications and nondestructive three-dimensional imaging. Journal of Physical Organic Chemistry 2000, 13 (12), 837-849.

43. Kim, R. H.; Lee, K.-S., 3D Stereolithography by Using Two-Photon Photopolymerization. Macromolecular Symposia 2010, 298 (1), 25-33.

44. Wang, I.; Bouriau, M.; Baldeck, P. L.; Martineau, C.; Andraud, C., Three-dimensional microfabrication by twophoton-initiated polymerization with a low-cost microlaser. Opt. Lett. 2002, 27 (15), 1348-1350.

45. Li, Z.; Hu, P.; Zhu, J.; Gao, Y.; Xiong, X.; Liu, R., Conjugated Carbazole-Based Schiff Bases as Photoinitiators: From Facile Synthesis to Efficient Two-Photon Polymerization. Journal of Polymer Science Part A: Polymer Chemistry 2018, 56 (23), 2692-2700.

46. Xing, J.-F.; Zheng, M.-L.; Chen, W.-Q.; Dong, X.-Z.; Takeyasu, N.; Tanaka, T.; Zhao, Z.-S.; Duan, X.-M.; Kawata, S., C $2 \mathrm{v}$ symmetrical two-photon polymerization initiators with anthracene core: synthesis, optical and initiating properties. Physical Chemistry Chemical Physics 2012, 14 (45), 1578515792.

47. Wang, X.; Jin, F.; Zhang, W.; Tao, X.; Duan, X.-M.; Jiang M., Novel D- $\pi 3-(A) 1-3$ multibranched chromophores as an efficient two-photon-induced polymerization initiator. Dyes and Pigments 2011, 88 (1), 57-64.

48. $\quad$ Li, Z.; Torgersen, J.; Ajami, A.; Mühleder, S.; Qin, X.; Husinsky, W.; Holnthoner, W.; Ovsianikov, A.; Stampfl, J.; Liska, R., Initiation efficiency and cytotoxicity of novel water-soluble two-photon photoinitiators for direct 3D microfabrication of hydrogels. RSC Advances 2013, 3 (36), 15939-15946.

49. Whitby, R.; Kay, A.; Simpson, M. C., Chapter 2.2 Triphenylamine two-photon photoinitiators for 3D laser microfabrication. In Three-Dimensional Microfabrication Using Two-Photon Polymerization (Second Edition), Baldacchini, T., Ed. William Andrew Publishing: 2020; pp 101-141.

50. Lemercier, G.; Martineau, C.; Mulatier, J.-C.; Wang, I.; Stéphan, O.; Baldeck, P.; Andraud, C., Analogs of Michler's ketone for two-photon absorption initiation of polymerization in the near infrared: synthesis and photophysical properties. New Journal of Chemistry 2006, 30 (11), 1606-1613.
51. For dipolar chromophores. For quadrupolar ones, selection rules being mutually exclusive in one and two-photon absorptions, lowest accesible two-photon transition takes place to the S2 excited state instead of the S1 so that the maximal two-photon absorption wavelength is significantly less than twice that of the maximal one-photon absorption wavelength

52. Beljonne, D.; Wenseleers, W.; Zojer, E.; Shuai, Z.; Vogel, H.; Pond, S. J. K.; Perry, J. W.; Marder, S. R.; Brédas, J.-L., Role of Dimensionality on the Two-Photon Absorption Response of Conjugated Molecules: The Case of Octupolar Compounds. Advanced Functional Materials 2002, 12 (9), 631641.

53. Kamada, K.; Ohta, K.; Iwase, Y.; Kondo, K., Two-photon absorption properties of symmetric substituted diacetylene: drastic enhancement of the cross section near the one-photon absorption peak. Chemical Physics Letters 2003, 372 (3), 386393.

54. Kogej, T.; Beljonne, D.; Meyers, F.; Perry, J. W.; Marder, S. R.; Brédas, J. L., Mechanisms for enhancement of two-photon absorption in donor-acceptor conjugated chromophores. Chemical Physics Letters 1998, 298 (1), 1-6.

55. Kamada, K.; Ohta, K.; Kubo, T.; Shimizu, A.; Morita, Y.; Nakasuji, K.; Kishi, R.; Ohta, S.; Furukawa, S.-i.; Takahashi, H.; Nakano, M., Strong Two-Photon Absorption of Singlet Diradical Hydrocarbons. Angewandte Chemie International Edition 2007, 46 (19), 3544-3546.

56. Drobizhev, M.; Karotki, A.; Kruk, M.; Rebane, A., Resonance enhancement of two-photon absorption in porphyrins. Chemical Physics Letters 2002, 355 (1), 175-182.

57. Ohira, S.; Rudra, I.; Schmidt, K.; Barlow, S.; Chung, S.J.; Zhang, Q.; Matichak, J.; Marder, S. R.; Brédas, J.-L., Electronic and Vibronic Contributions to Two-Photon Absorption in Donor-Acceptor-Donor Squaraine Chromophores. Chemistry A European Journal 2008, 14 (35), 11082-11091.

58. Collins, H. A.; Khurana, M.; Moriyama, E. H.; Mariampillai, A.; Dahlstedt, E.; Balaz, M.; Kuimova, M. K.; Drobizhev, M.; Yang, V. X. D.; Phillips, D.; Rebane, A.; Wilson, B. C.; Anderson, H. L., Blood-vessel closure using photosensitizers engineered for two-photon excitation. Nature Photonics 2008, 2 (7), 420-424.

59. Jin, M.; Xie, J.; Malval, J.-P.; Spangenberg, A.; Soppera, O.; Versace, D.-L.; Leclerc, T.; Pan, H.; Wan, D.; Pu, H.; Baldeck, P.; Poizat, O.; Knopf, S., Two-photon lithography in visible and NIR ranges using multibranched-based sensitizers for efficient acid generation. Journal of Materials Chemistry C 2014, 2 (35), 7201-7215.

60. Ovsianikov, A.; Viertl, J.; Chichkov, B.; Oubaha, M.; MacCraith, B.; Sakellari, I.; Giakoumaki, A.; Gray, D.; Vamvakaki, M.; Farsari, M.; Fotakis, C., Ultra-Low Shrinkage Hybrid Photosensitive Material for Two-Photon Polymerization Microfabrication. ACS Nano 2008, 2 (11), 2257-2262.

61. Sakellari, I.; Kabouraki, E.; Gray, D.; Purlys, V.; Fotakis, C.; Pikulin, A.; Bityurin, N.; Vamvakaki, M.; Farsari, M., Diffusion-Assisted High-Resolution Direct Femtosecond Laser Writing. ACS Nano 2012, 6 (3), 2302-2311.

62. Rajamanickam, V. P.; Ferrara, L.; Toma, A.; Proietti Zaccaria, R.; Das, G.; Di Fabrizio, E.; Liberale, C., Suitable photoresists for two-photon polymerization using femtosecond fiber lasers. Microelectronic Engineering 2014, 121, 135-138.

63. Fischer, J.; von Freymann, G.; Wegener, M., The Materials Challenge in Diffraction-Unlimited Direct-LaserWriting Optical Lithography. Advanced Materials 2010, 22 (32), 3578-3582. 
64. Tsutsumi, N.; Hirota, J.; Kinashi, K.; Sakai, W., Direct laser writing for micro-optical devices using a negative photoresist. Opt. Express 2017, 25 (25), 31539-31551.

65. Zhou, R.; Sun, X.; Mhanna, R.; Malval, J.-P.; Jin, M.; Pan, H.; Wan, D.; Morlet-Savary, F.; Chaumeil, H.; Joyeux, C., Wavelength-Dependent, Large-Amplitude Photoinitiating Reactivity within a Carbazole-Coumarin Fused Oxime Esters Series. ACS Applied Polymer Materials 2020.

66. Mettra, B.; Liao, Y. Y.; Gallavardin, T.; Armagnat, C.; Pitrat, D.; Baldeck, P.; Le Bahers, T.; Monnereau, C.; Andraud, C., A combined theoretical and experimental investigation on the influence of the bromine substitution pattern on the photophysics of conjugated organic chromophores. Physical Chemistry Chemical Physics 2018, 20 (5), 3768-3783.

67. Lanoë, P.-H.; Gallavardin, T.; Dupin, A.; Maury, O.; Baldeck, P. L.; Lindgren, M.; Monnereau, C.; Andraud, C., Influence of bromine substitution pattern on the singlet oxygen generation efficiency of two-photon absorbing chromophores. Organic \& Biomolecular Chemistry 2012, 10 (31), 6275-6278.

68. Kamada, K.; Iwase, Y.; Sakai, K.; Kondo, K.; Ohta, K., Cationic Two-Photon Absorption Chromophores with Doubleand Triple-Bond Cores in Symmetric/Asymmetric Arrangements. The Journal of Physical Chemistry C 2009, 113 (27), 11469-11474.

69. Kennedy, S. M.; Lytle, F. E., p-Bis(omethylstyryl)benzene as a power-squared sensor for twophoton absorption measurements between 537 and $694 \mathrm{~nm}$. Analytical Chemistry 1986, 58 (13), 2643-2647.

70. $\quad$ Frisch, M. J.; Trucks, G. W.; Schlegel, H. B.; Scuseria, G. E.; Robb, M. A.; Cheeseman, J. R.; Scalmani, G.; Barone, V.; Petersson, G. A.; Nakatsuji, H.; Li, X.; Caricato, M.; Marenich, A. V.; Bloino, J.; Janesko, B. G.; Gomperts, R.; Mennucci, B.; Hratchian, H. P.; Ortiz, J. V.; Izmaylov, A. F.; Sonnenberg, J. L.; Williams; Ding, F.; Lipparini, F.; Egidi, F.; Goings, J.; Peng, B.; Petrone, A.; Henderson, T.; Ranasinghe, D.; Zakrzewski, V. G.; Gao, J.; Rega, N.; Zheng, G.; Liang, W.; Hada, M.; Ehara, M.; Toyota, K.; Fukuda, R.; Hasegawa, J.; Ishida, M.; Nakajima, T.; Honda, Y.; Kitao, O.; Nakai, H.; Vreven, T.; Throssell, K.; Montgomery Jr., J. A.; Peralta, J. E.; Ogliaro, F.; Bearpark, M. J.; Heyd, J. J.; Brothers, E. N.; Kudin, K. N.; Staroverov, V. N.; Keith, T. A.; Kobayashi, R.; Normand, J.; Raghavachari, K.; Rendell, A. P.; Burant, J. C.; Iyengar, S. S.; Tomasi, J.; Cossi, M.; Millam, J. M.; Klene, M.; Adamo, C.; Cammi, R.; Ochterski, J. W.; Martin, R. L.; Morokuma, K.; Farkas, O.; Foresman, J. B.; Fox, D. J. Gaussian 16 Rev. C.01, Wallingford, CT, 2016.

71. Marenich, A. V.; Ho, J.; Coote, M. L.; Cramer, C. J.; Truhlar, D. G., Computational electrochemistry: prediction of liquid-phase reduction potentials. Physical Chemistry Chemical Physics 2014, 16 (29), 15068-15106.

72. Allen, N. S.; Catalina, F.; Green, P. N.; Green, W. A., Photochemistry of thioxanthones-I. Spectroscopic and flash photolysis study on oil soluble structures. European Polymer Journal 1985, 21 (10), 841-848.

73. Gallavardin, T.; Armagnat, C.; Maury, O.; Baldeck, P. L.; Lindgren, M.; Monnereau, C.; Andraud, C., An improved singlet oxygen sensitizer with two-photon absorption and emission in the biological transparency window as a result of ground state symmetry-breaking. Chemical Communications 2012, 48 (11), 1689-1691.

74. Yang, W. J.; Kim, C. H.; Jeong, M.-Y.; Lee, S. K.; Piao, M. J.; Jeon, S.-J.; Cho, B. R., Synthesis and Two-Photon Absorption Properties of 9,10-Bis(arylethynyl)anthracene Derivatives. Chemistry of Materials 2004, 16 (14), 2783-2789.
75. Morisue, M.; Ogawa, K.; Kamada, K.; Ohta, K.; Kobuke, Y., Strong two-photon and three-photon absorptions in the antiparallel dimer of a porphyrin-phthalocyanine tandem. Chemical Communications 2010, 46 (12), 2121-2123.

76. Garoni, E.; Nisic, F.; Colombo, A.; Fantacci, S.; Griffini, G.; Kamada, K.; Roberto, D.; Dragonetti, C., Perylenetetracarboxy-3,4:9,10-diimide derivatives with large two-photon absorption activity. New Journal of Chemistry 2019, 43 (4), 1885-1893.

77. Janzen, E. G.; Krygsman, P. H.; Lindsay, D. A.; Haire, D. L., Detection of alkyl, alkoxyl, and alkyperoxyl radicals from the thermolysis of azobis(isobutyronitrile) by ESR/spin trapping. Evidence for double spin adducts from liquid-phase chromatography and mass spectroscopy. Journal of the American Chemical Society 1990, 112 (23), 8279-8284.

78. Pryor, W. A.; Govindan, C. K.; Church, D. F., Mechanism of ozonolysis of acetylenes: evidence for a free-radical pathway for the decomposition of intermediates. Journal of the American Chemical Society 1982, 104 (26), 7563-7566.

79. Buettner, G. R., Spin Trapping: ESR parameters of spin adducts 1474 1528V. Free Radical Biology and Medicine 1987, 3 (4), 259-303.

80. Ding, G.; Jing, C.; Qin, X.; Gong, Y.; Zhang, X.; Zhang, S.; Luo, Z.; Li, H.; Gao, F., Conjugated dyes carrying N, Ndialkylamino and ketone groups: One-component visible light Norrish type II photoinitiators. Dyes and Pigments 2017, 137, 456-467.

81. Dadashi-Silab, S.; Doran, S.; Yagci, Y., Photoinduced Electron Transfer Reactions for Macromolecular Syntheses. Chemical Reviews 2016, 116 (17), 10212-10275.

82. Zhang, J.; Zivic, N.; Dumur, F.; Xiao, P.; Graff, B.; Fouassier, J. P.; Gigmes, D.; Lalevée, J., Naphthalimide-Tertiary Amine Derivatives as Blue-Light-Sensitive Photoinitiators. ChemPhotoChem 2018, 2 (6), 481-489.

83. Tehfe, M.-A.; Zein-Fakih, A.; Lalevée, J.; Dumur, F.; Gigmes, D.; Graff, B.; Morlet-Savary, F.; Hamieh, T.; Fouassier, J.P., New pyridinium salts as versatile compounds for dye sensitized photopolymerization. European Polymer Journal 2013, 49 (2), 567-574.

84. Criqui, A.; Lalevée, J.; Allonas, X.; Fouassier, J.-P., Electron Spin Resonance Spin Trapping Technique: Application to the Cleavage Process of Photoinitiators. Macromolecular Chemistry and Physics 2008, 209 (21), 2223-2231.

85. Migita, C. T.; Migita, K., Spin Trapping of the Nitrogencentered Radicals. Characterization of the DMPO/DEPMPO Spin Adducts. Chemistry Letters 2003, 32 (5), 466-467.

86. Podsiadły, R.; Podemska, K.; Szymczak, A. M., Novel visible photoinitiators systems for free-radical/cationic hybrid photopolymerization. Dyes and Pigments 2011, 91 (3), 422426.

87. Hu, J.; Wang, J.; Nguyen, T. H.; Zheng, N., The chemistry of amine radical cations produced by visible light photoredox catalysis. Beilstein Journal of Organic Chemistry 2013, 9, 19772001

88. M'Halla, F.; Pinson, J.; Saveant, J. M., Hydrogen atom abstraction and solvent involvement in the electrochemistry of haloaromatics: Reduction of 4-bromobenzophenone in dimethylsulfoxide. Journal of Electroanalytical Chemistry and Interfacial Electrochemistry 1978, 89 (2), 347-361.

89. Lemee, V.; Burget, D.; Jacques, P.; Fouassier, J. P., On the interaction mechanism of radical photoinitiators with monomers. Journal of Polymer Science Part A: Polymer Chemistry 2000, 38 (10), 1785-1794. 
90. Kabatc, J.; Kostrzewska, K.; Kozak, M.; Balcerak, A., Visible light photoinitiating systems based on squaraine dye: kinetic, mechanistic and laser flash photolysis studies. RSC Advances 2016, 6 (105), 103851-103863.

91. Al Mousawi, A.; Dumur, F.; Garra, P.; Toufaily, J.; Hamieh, T.; Graff, B.; Gigmes, D.; Fouassier, J. P.; Lalevée, J., Carbazole Scaffold Based Photoinitiator/Photoredox Catalysts: Toward New High Performance Photoinitiating Systems and Application in LED Projector 3D Printing Resins. Macromolecules 2017, 50 (7), 2747-2758.

92. Mitterbauer, M.; Knaack, P.; Naumov, S.; Markovic, M.; Ovsianikov, A.; Moszner, N.; Liska, R., Acylstannanes: Cleavable and Highly Reactive Photoinitiators for Radical Photopolymerization at Wavelengths above $500 \mathrm{~nm}$ with Excellent Photobleaching Behavior. Angewandte Chemie International Edition 2018, 57 (37), 12146-12150.

93. Zhao, J.; Lalevée, J.; Lu, H.; MacQueen, R.; Kable, S. H.; Schmidt, T. W.; Stenzel, M. H.; Xiao, P., A new role of curcumin: as a multicolor photoinitiator for polymer fabrication under household UV to red LED bulbs. Polymer Chemistry 2015, 6 (28), 5053-5061.

94. Tehfe, M.-A.; Dumur, F.; Graff, B.; Gigmes, D.; Fouassier, J.-P.; Lalevée, J., Blue-to-Red Light Sensitive PushPull Structured Photoinitiators: Indanedione Derivatives for Radical and Cationic Photopolymerization Reactions. Macromolecules 2013, 46 (9), 3332-3341.

95. Al Mousawi, A.; Poriel, C.; Dumur, F.; Toufaily, J.; Hamieh, T.; Fouassier, J. P.; Lalevée, J., Zinc Tetraphenylporphyrin as High Performance Visible Light Photoinitiator of Cationic Photosensitive Resins for LED Projector 3D Printing Applications. Macromolecules 2017, 50 (3), 746-753.
96. In the case of BDEBP, at the power used in this comparative study, reaction proceeds to fast to reliably fit the initial stage of the reaction. A first order is however seen when decreasing incident light power (data not included).

97. Suppan, P., Photoreactivity of Michler's ketone in solution. Journal of the Chemical Society, Faraday Transactions 1: Physical Chemistry in Condensed Phases 1975, 71 (0), 539547.

98. Gan, Z.; Cao, Y.; Evans, R.; Gu, M., Three-dimensional deep sub-diffraction optical beam lithography with $9 \mathrm{~nm}$ feature size. Nature communications 2013, 4, 2061.

99. Prabhakaran, P.; Son, Y.; Ha, C.-W.; Park, J.-J.; Jeon, S.; Lee, K.-S., Optical Materials Forming Tightly Polymerized Voxels during Laser Direct Writing. Advanced Engineering Materials 2018, 20 (10), 1800320.

100. Saha, S. K.; Wang, D.; Nguyen, V. H.; Chang, Y.; Oakdale, J. S.; Chen, S.-C., Scalable submicrometer additive manufacturing. Science 2019, 366 (6461), 105-109.

101. Fischer, J.; Mueller, J. B.; Kaschke, J.; Wolf, T. J. A.; Unterreiner, A.-N.; Wegener, M., Three-dimensional multiphoton direct laser writing with variable repetition rate. $O p t$. Express 2013, 21 (22), 26244-26260.

102. Dong, X.-Z.; Zhao, Z.-S.; Duan, X.-M., Improving spatial resolution and reducing aspect ratio in multiphoton polymerization nanofabrication. Applied Physics Letters 2008, $92(9), 091113$.

103. Martineau, C.; Anémian, R.; Andraud, C.; Wang, I.; Bouriau, M.; Baldeck, P. L., Efficient initiators for two-photon induced polymerization in the visible range. Chemical Physics Letters 2002, 362 (3), 291-295. 


\section{Supporting Information}

\section{Polymerization Photoinitiators with Near-Resonance Enhanced Two-Photon Absorption Cross- Section towards High-Resolution Photoresists with Improved Sensitivity}

Caroline Arnoux, ${ }^{\dagger}$ Tatsuki Konishi, ${ }^{,}$Eric-Aimé Poutougnigni, ${ }^{\dagger}$ Emma Van Elslande, ${ }^{\dagger}$ Jean-Christophe Mulatier, ${ }^{\dagger}$ Lhoussain Khrouz, ${ }^{\dagger}$ Christophe Bucher, ${ }^{\dagger}$ Elise Dumont, ${ }^{\dagger}$ Kenji Kamada, ${ }^{\ddagger}$ Patrice Baldeck, ${ }^{\dagger}$ Chantal Andraud ${ }^{\dagger}$ Akos Banyasz, ${ }^{\dagger}$ Cyrille Monnereau ${ }^{\dagger}$

† Univ. Lyon, ENS Lyon, CNRS, Université Lyon 1, Laboratoire de Chimie, UMR 5182, 46 Allée d'Italie, 69364 Lyon, France.

‡ Nanomaterials Research Institute (NMRI), National Institute of Advanced Industrial Science and Technology (AIST), 1-8-31 Midorigaoka, Ikeda, Osaka, 563-8577 Japan

\section{Contents}

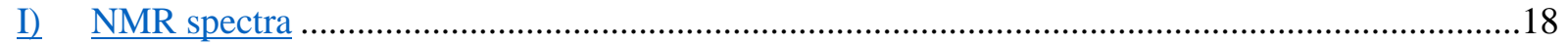

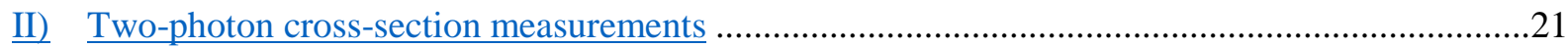

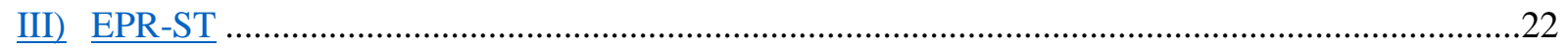

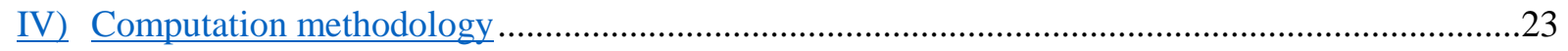

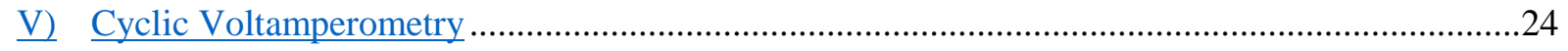

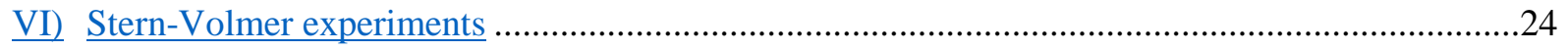

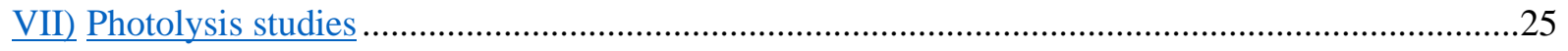

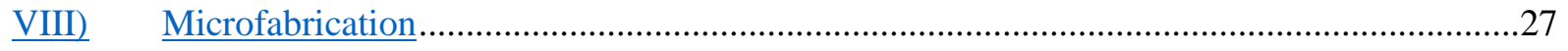

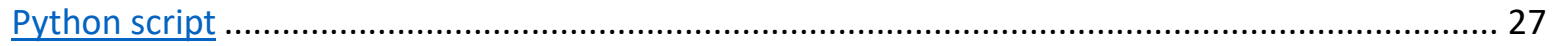

Evolution of the line widths and heights versus laser power .................................................... 27

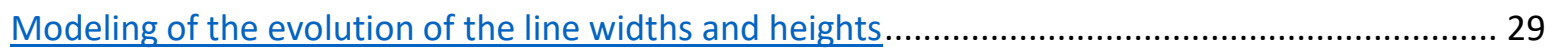


I) NMR spectra

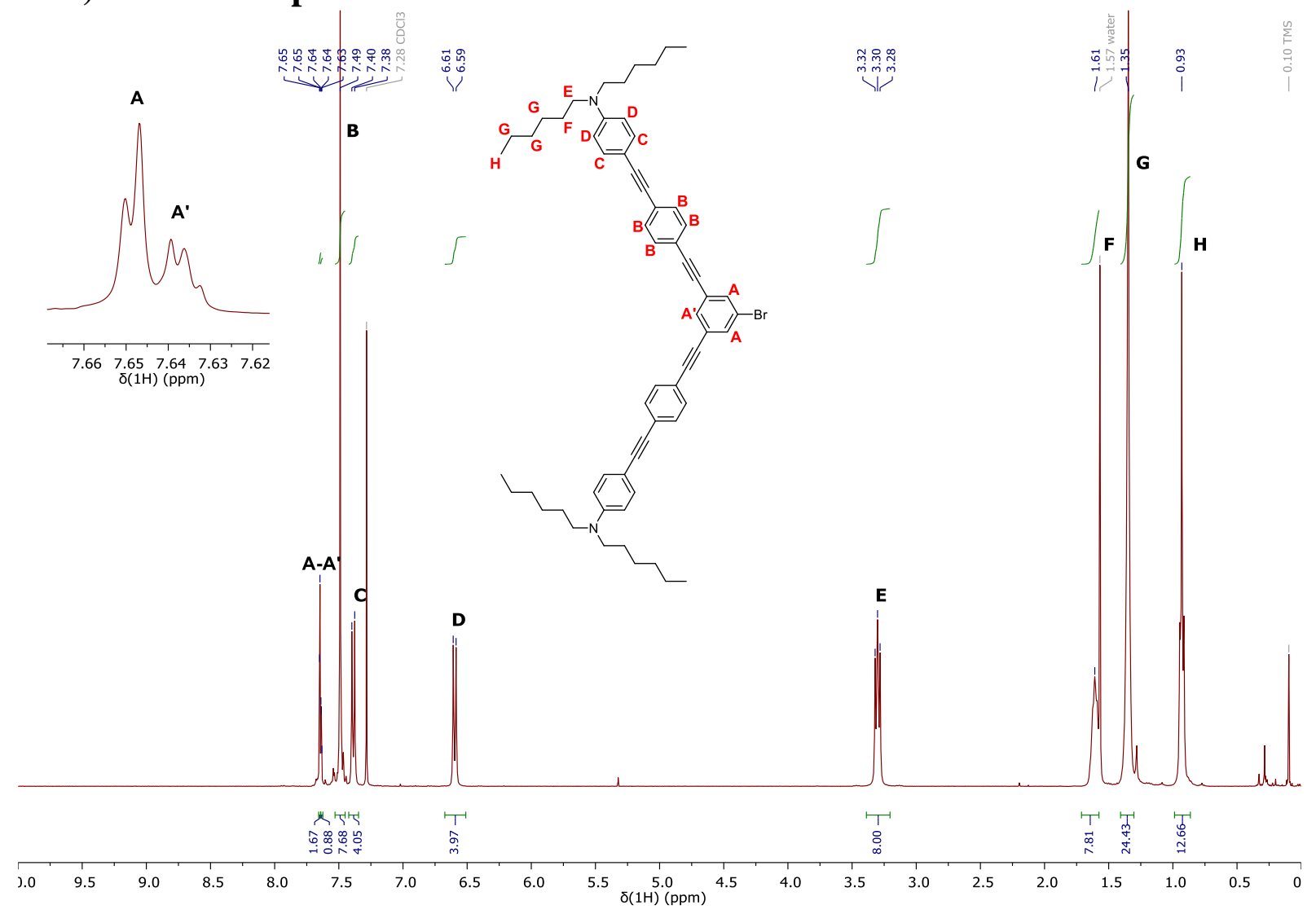



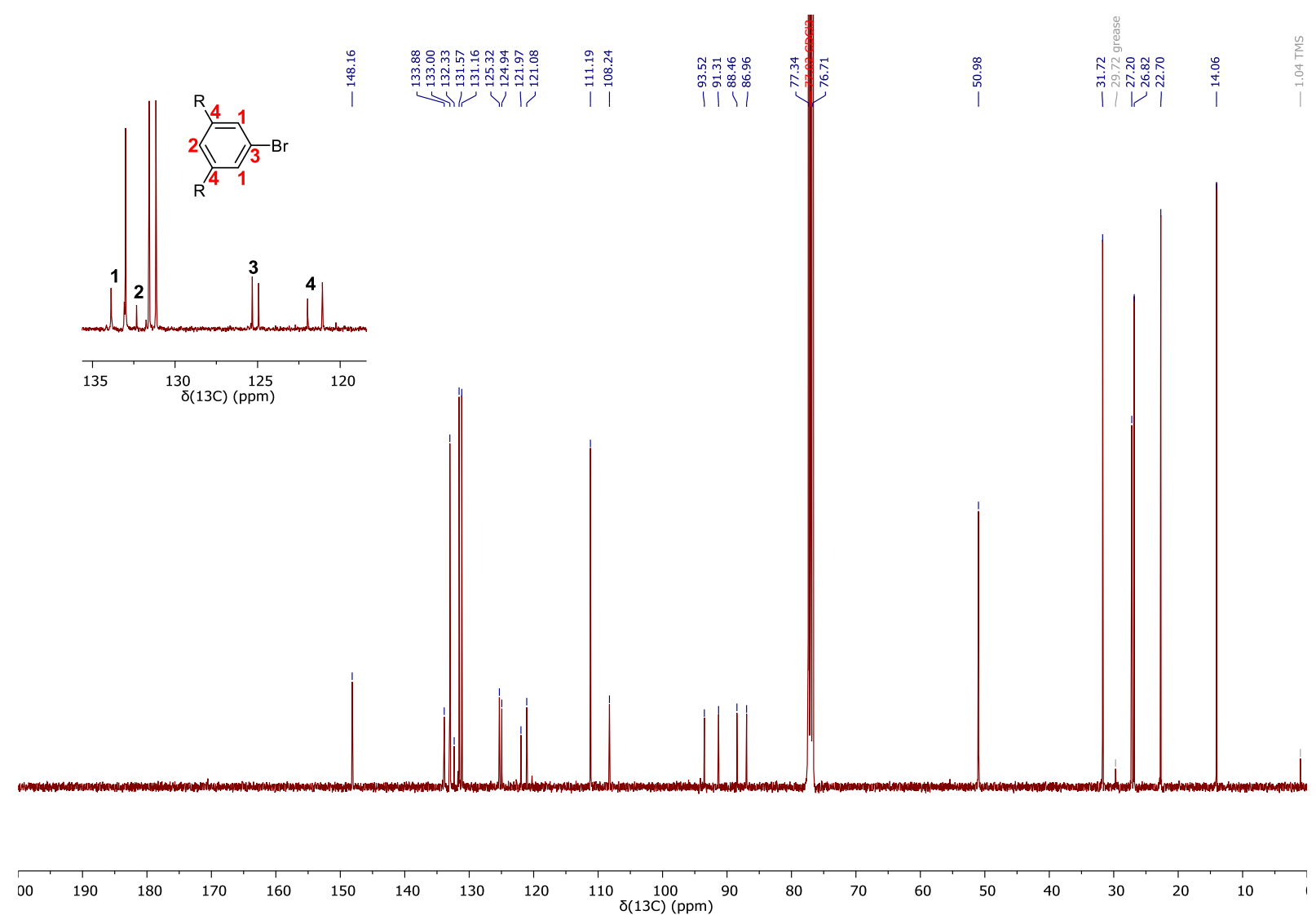

Figure S11. ${ }^{1} \mathrm{H}$ and ${ }^{13} \mathrm{C}$ NMR spectra of V-Shape in $\mathrm{CDCl}_{3}$.

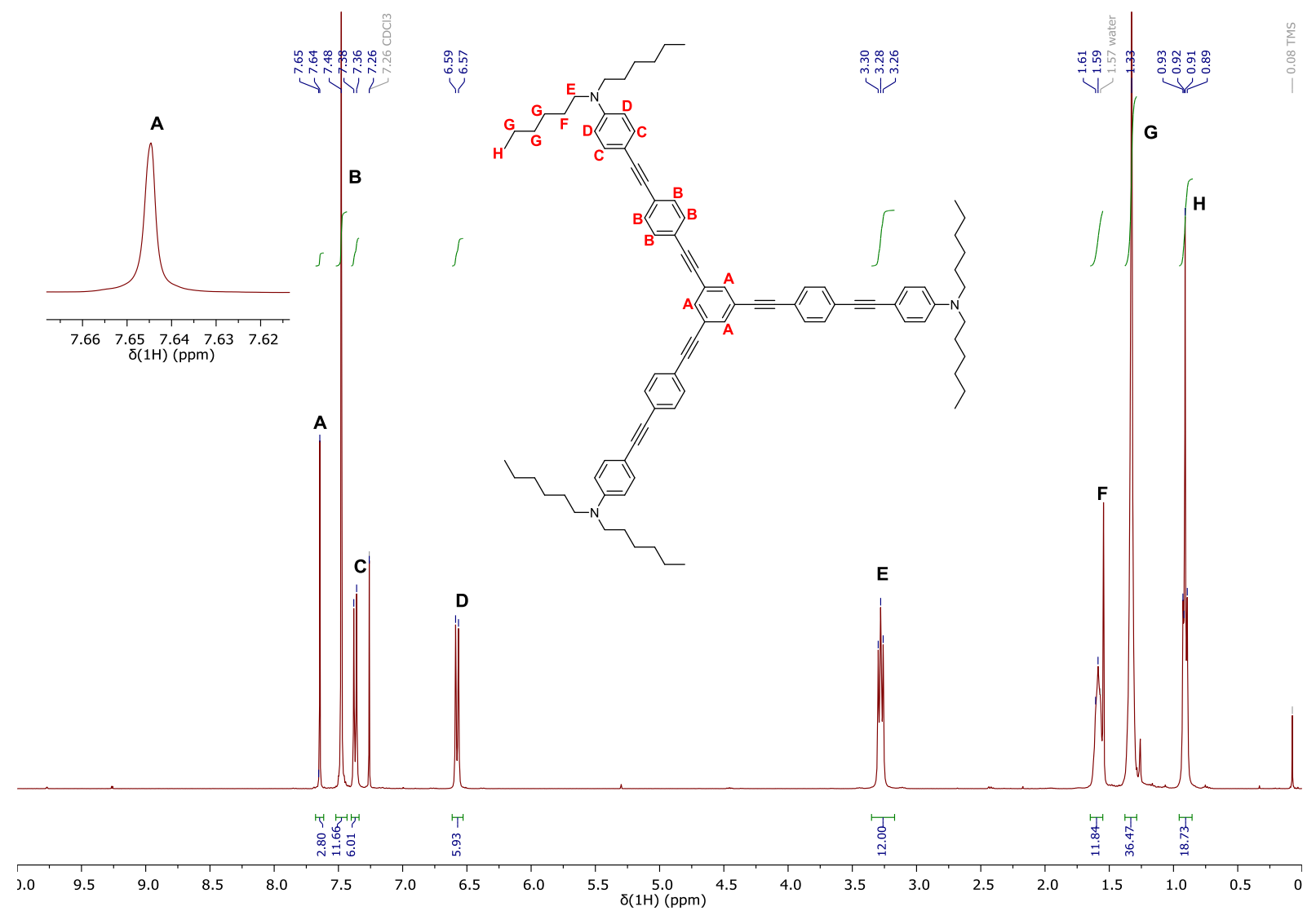




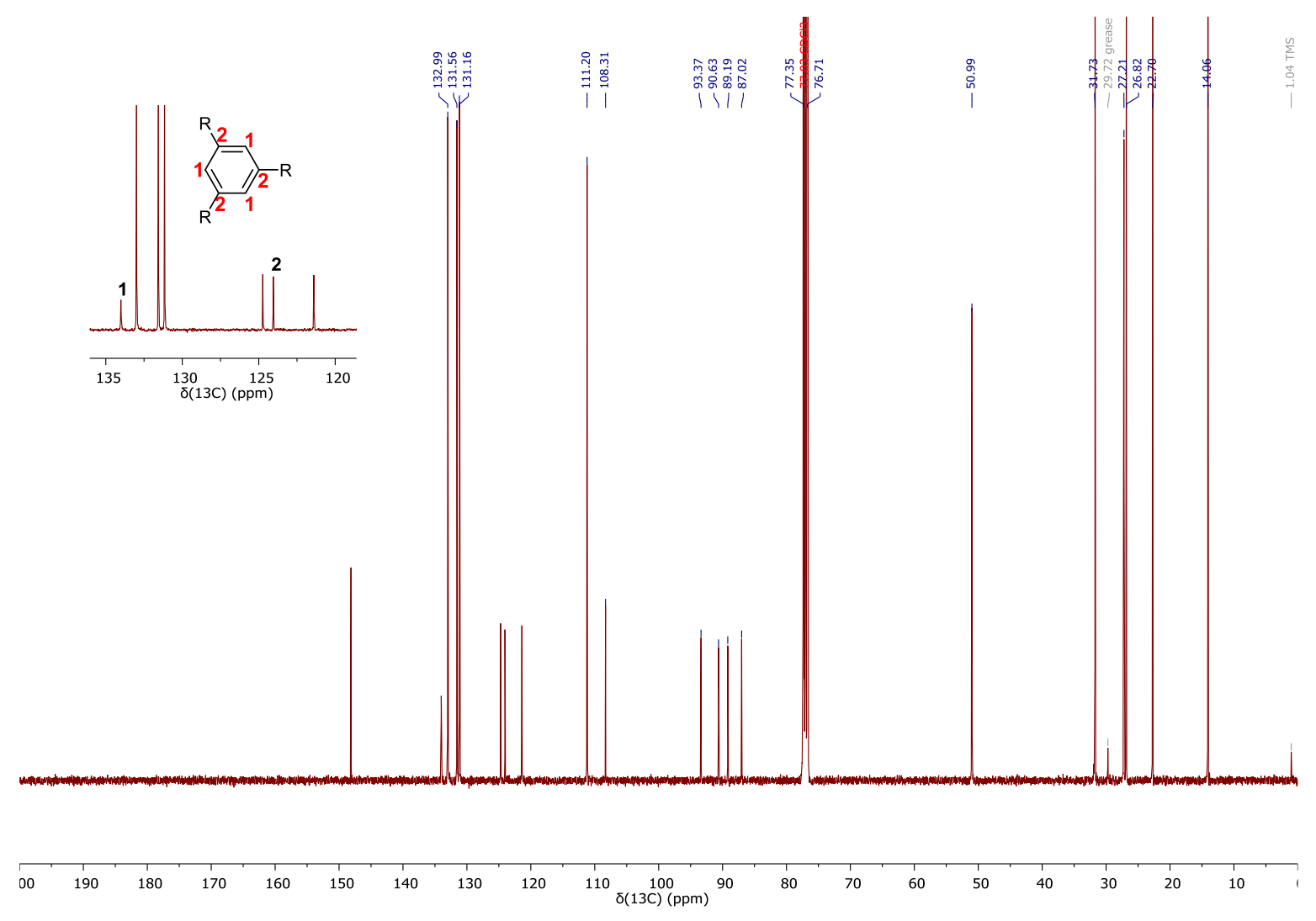

Figure S12. ${ }^{1} \mathrm{H}$ and ${ }^{13} \mathrm{C}$ NMR spectra of Oct in $\mathrm{CDCl}_{3}$. 
II) Two-photon cross-section measurements

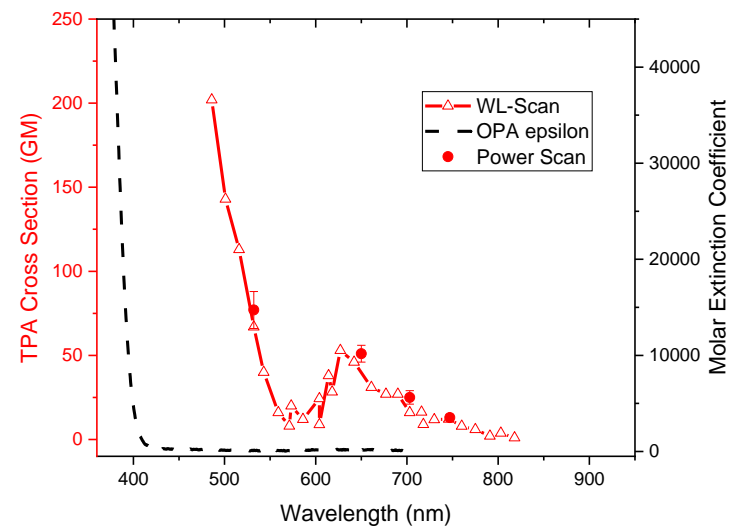

Figure S13. TPA spectra of BDEBP (red) in dichloromethane obtained by wavelength (WL)-scan measurement and power scan measurement for some selected wavelengths with a close-up on the chromophore's absorption tail (black dotted line).

\section{V-Shape}
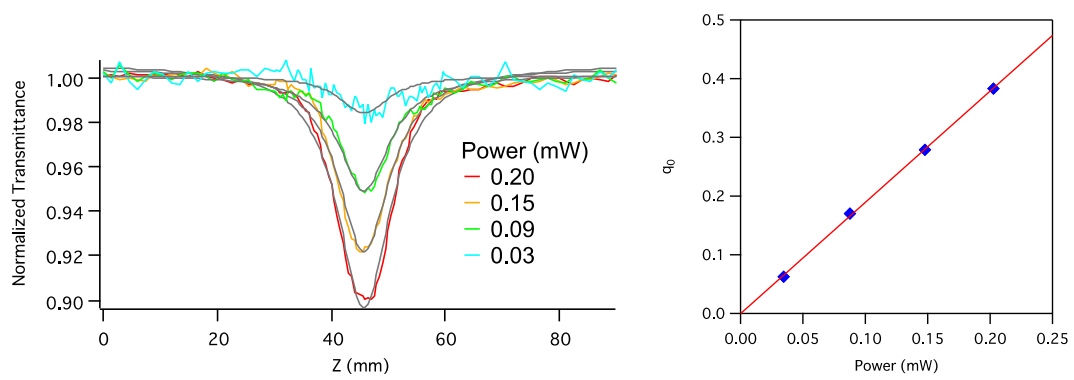

Oct
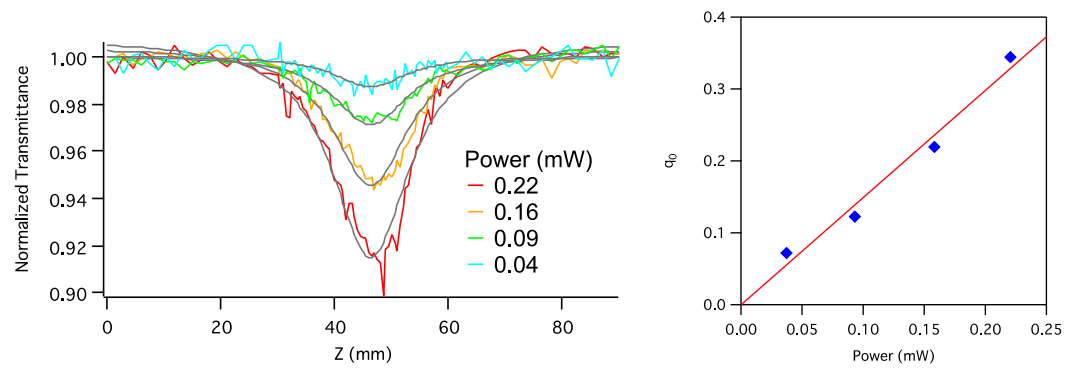

\section{BDEBP}
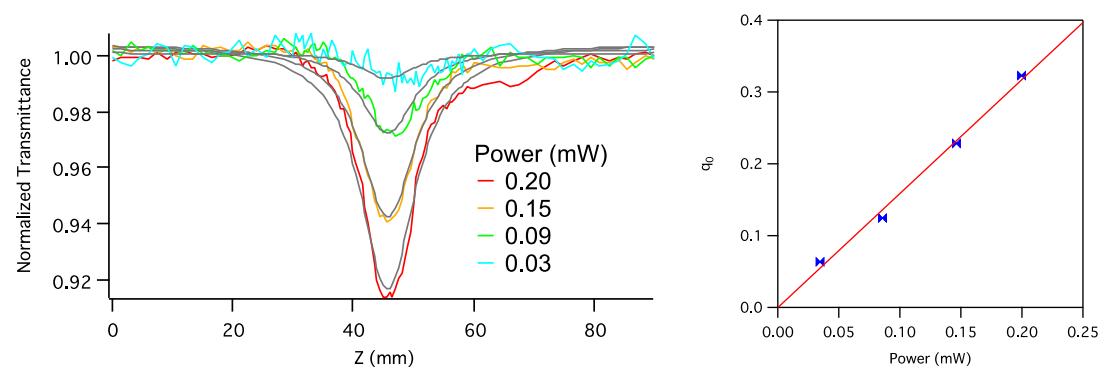

Figure S14. Open-aperture z-scan traces by power-scan at $532 \mathrm{~nm}$ and corresponding plot of the two-photon absorbance $q_{0} \mathrm{vs.}$ the incident power. 

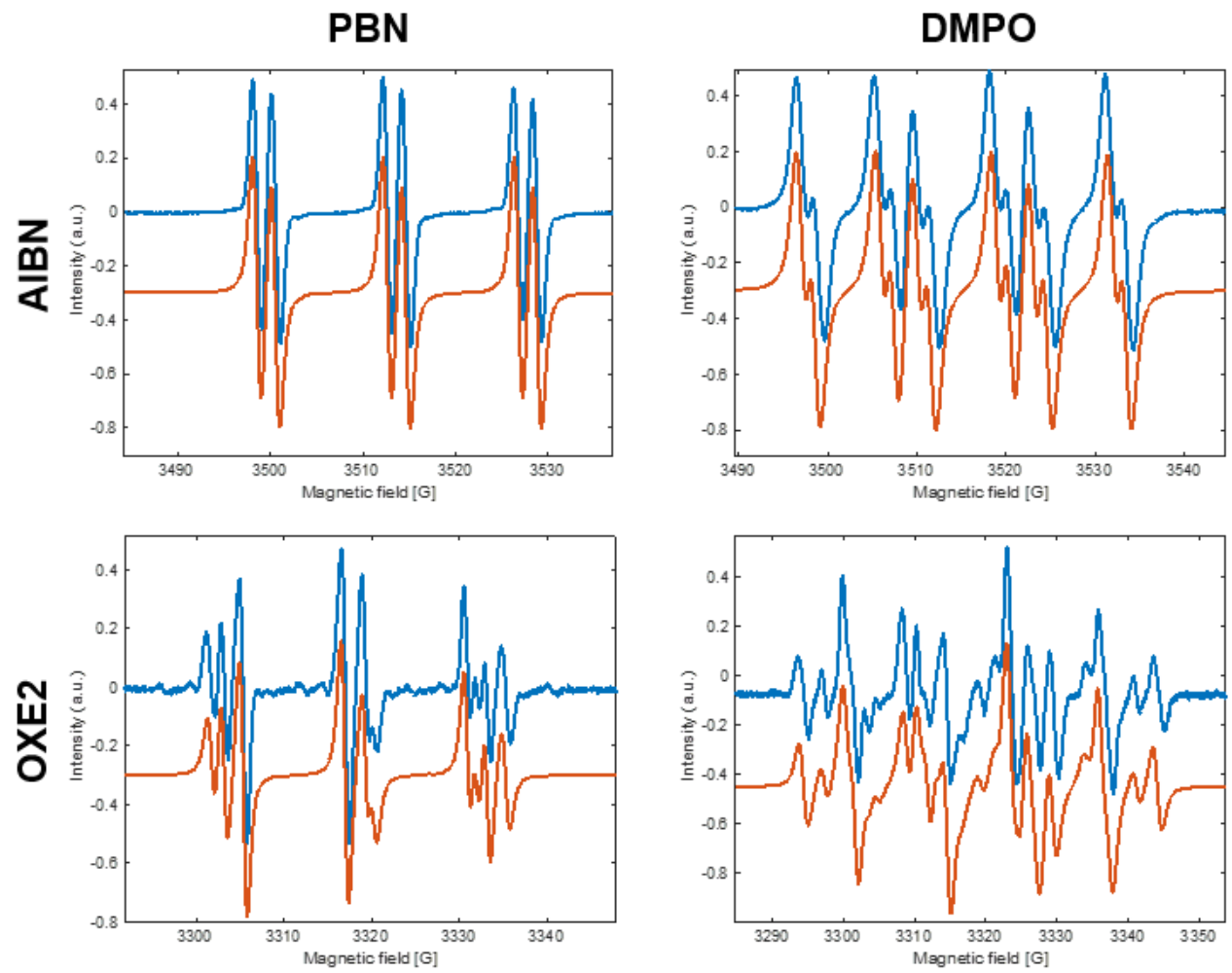

Figure S15. EPR spectra of spin trap adducts generated after irradiation of the AIBN and OXE2 at $365 \mathrm{~nm}$ in presence of PBN or DMPO in dichloromethane. Experimental spectra (blue) and simulated spectra (orange). 


\section{IV) Computation methodology}

Density functional theory (DFT) calculations were performed at the M06-HF/6-31G(d,p) level of theory on V-Shape to evaluate the relative energies for the neutral, radical anionic and radical cationic species. Results with inclusion of dichloromethane (IEF-PCM formalism) are shown in Figure S16.

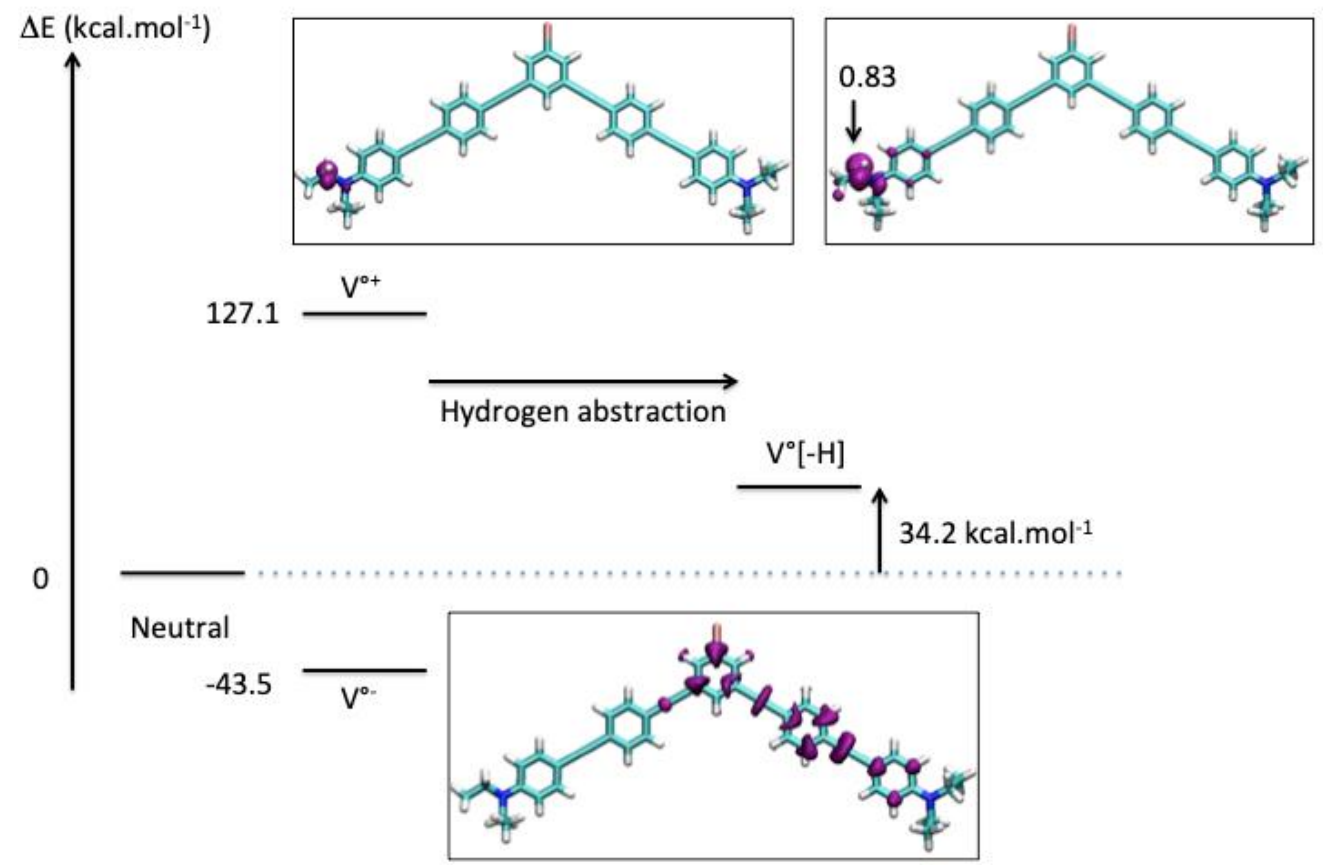

Figure S16. Energies of V-Shape, with licorice representations of the stationary point on the potential energy surface. Isodensities are plotted with a contour value of 0.05 .

Absolute redox potentials were estimated at $298 \mathrm{~K}$, after frequencies calculations according to the computational protocol proposed by Truhlar and coworkers. ${ }^{1}$ Frequencies were computed within the harmonic approximation. The difference of redox potentials is estimated to $2.63 \mathrm{~V}$, in neat agreement with the experimental data. The inspection of spin densities indicates a more delocalized, hence more stable, anionic radical compared to the intrinsically unstable cation. 


\section{V) Cyclic Voltamperometry}

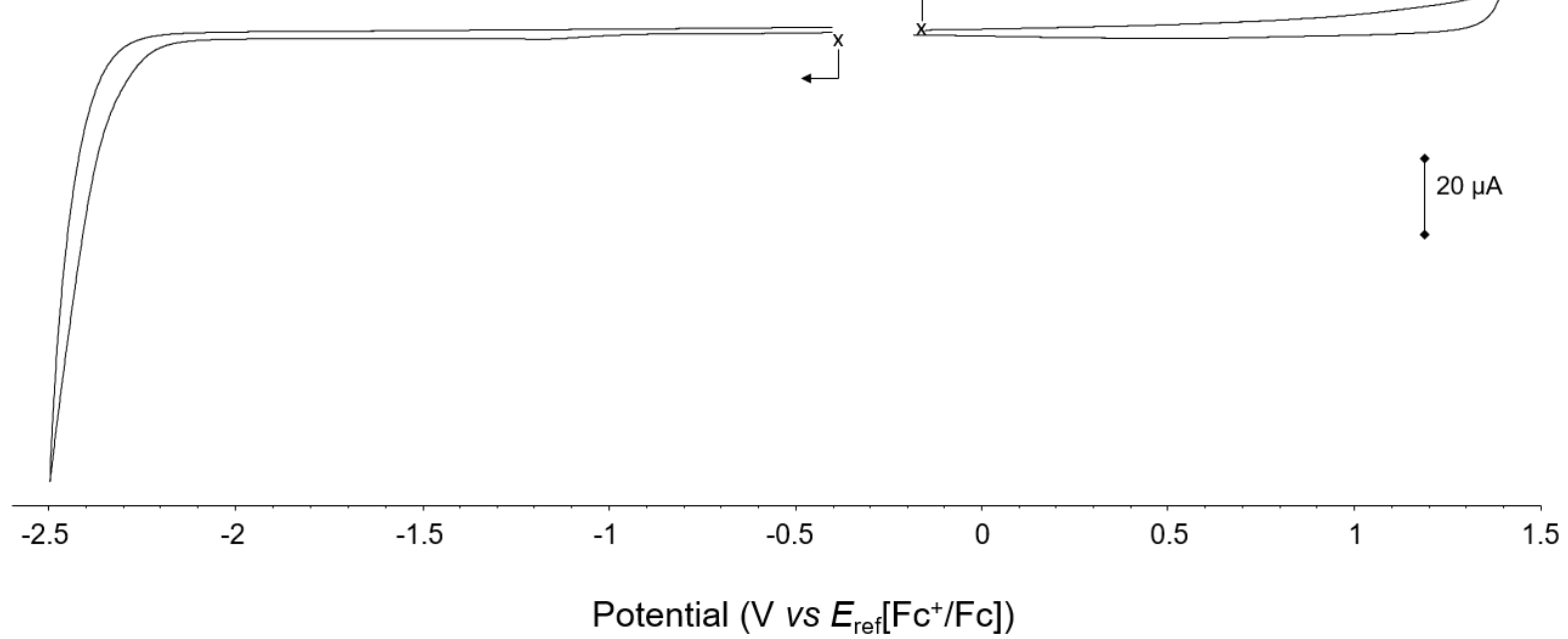

Figure S17. Cyclic voltammogram of DPPHA monomer $(1 \mathrm{mmol} / \mathrm{L})$ in dichloromethane in the presence of tetra-n-butylammonium perchlorate $(0.1 \mathrm{~mol} / \mathrm{L}), \mathrm{E}(\mathrm{V}) \mathrm{vs}$. $E_{\mathrm{ref}}\left[\mathrm{Fc} / \mathrm{Fc}^{+}\right]$, at a vitreous carbon working electrode $\emptyset=3 \mathrm{~mm}, 298 \mathrm{~K}, v=0.1 \mathrm{~V} / \mathrm{s}$.

\section{VI) Stern-Volmer experiments}
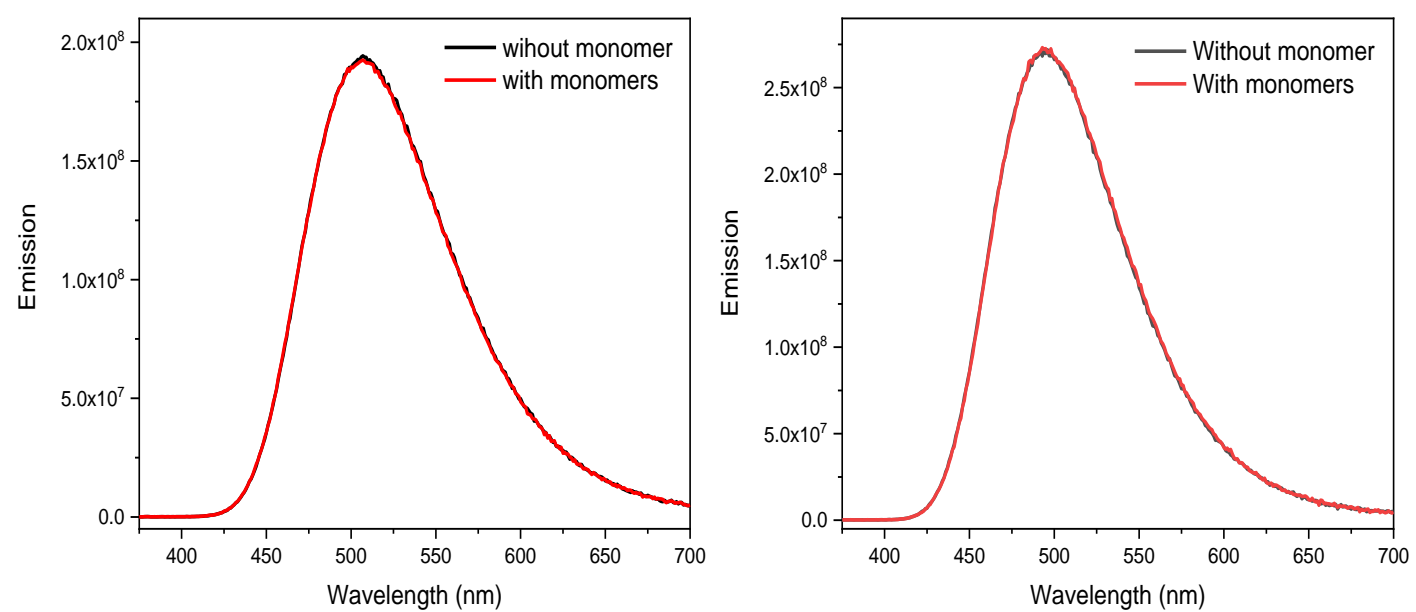

Figure S18. Emission spectra of $\boldsymbol{V}$-Shape $\left(2.66 \times 10^{-6} \mathrm{~mol} / \mathrm{L}\right.$, left $)$ and Oct $\left(2.08 \times 10^{-6} \mathrm{~mol} / \mathrm{L}\right.$, left $)$ with and without DPPHA $\left(4 \times 10^{-3}\right.$ mol/L). Solvent: dichloromethane, excitation at $\lambda_{e x}=370 \mathrm{~nm}$. 


\section{VII) Photolysis studies}
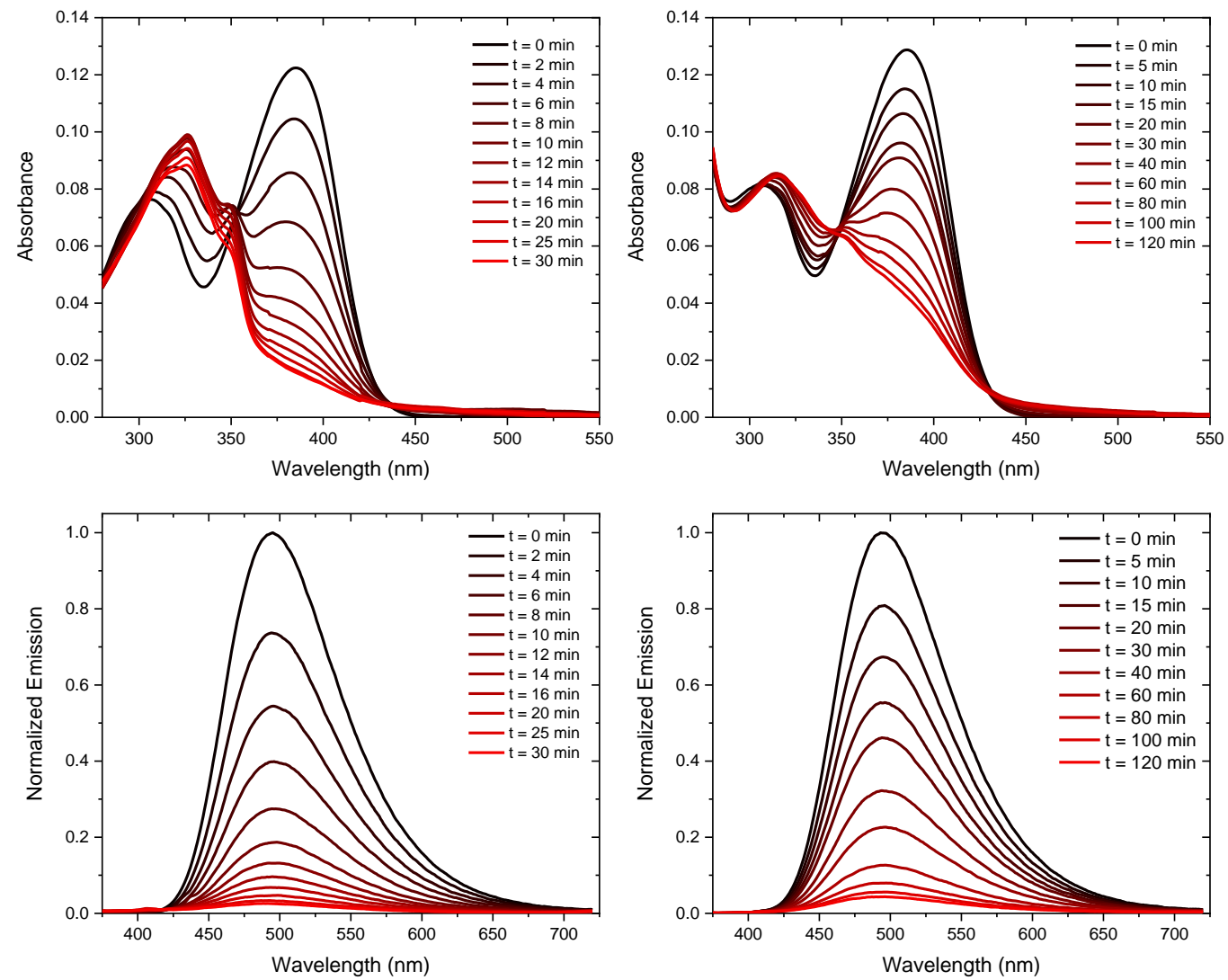

Figure S19. Photolysis studies (UV-Vis and emission) of Oct in degassed dichloromethane under $365 \mathrm{~nm}$ irradiation, $40 \mathrm{~mW}$ in absence or presence of EA. [Oct] $=8.6 \times 10-7 \mathrm{~mol} / \mathrm{L} ;[\mathrm{EA}]=0 \mathrm{~mol} / \mathrm{L}(\mathrm{left})$ and $7 \times 10-3 \mathrm{~mol} / \mathrm{L}(\mathrm{right})$. 


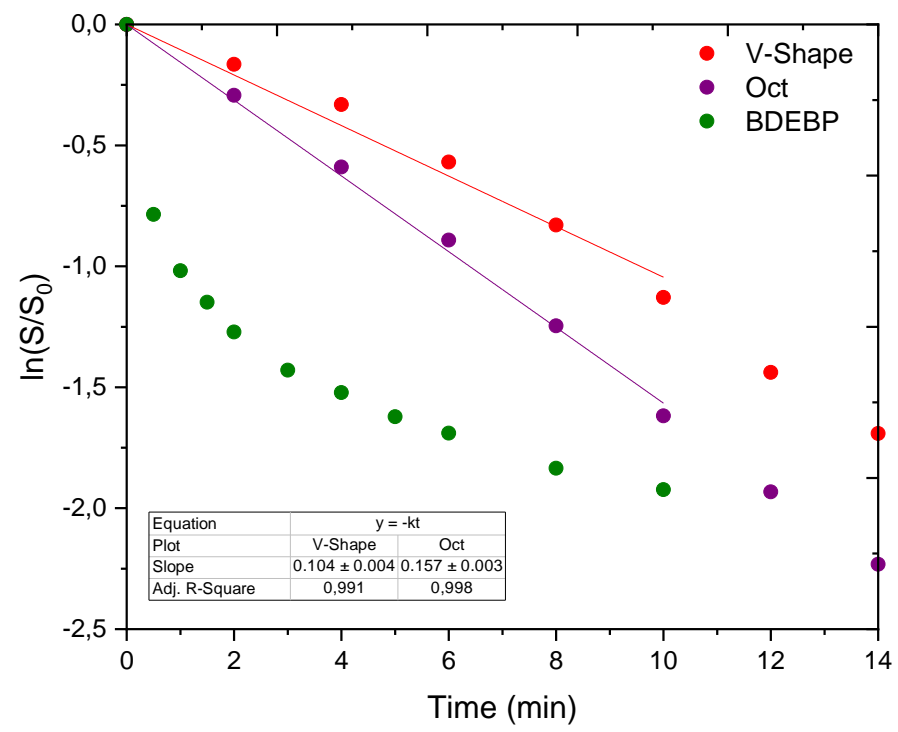

Figure S20. First order reaction fits for the photolysis of $\boldsymbol{V}$-Shape (red), Oct (purple) and unfitted scatter for BDEBP (green) in degassed dichloromethane under $365 \mathrm{~nm}$ irradiation, $40 \mathrm{~mW}$ in absence of EA at isoabsorbance concentration at $365 \mathrm{~nm}$ with estimated reaction rate constant $\mathrm{k}$. $[\mathrm{V}$-Shape $]=1.4 \times 10^{-6} \mathrm{~mol} / \mathrm{L} ;[\mathrm{Oct}]=8.6 \times 10^{-7} \mathrm{~mol} / \mathrm{L}$ and $[\mathrm{BDEBP}]=2.6 \times 10^{-6} \mathrm{~mol} / \mathrm{L}$.

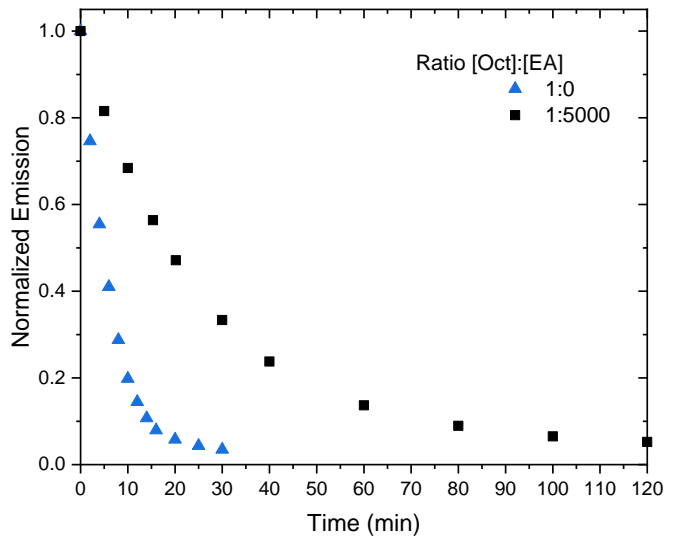

Figure S21. Photolysis of Oct in degassed dichloromethane under $365 \mathrm{~nm}$ irradiation, $40 \mathrm{~mW}$ in absence or presence of EA. [Oct] $=8.6 x$ $10^{-7} \mathrm{~mol} / \mathrm{L} ;[\mathrm{EA}]=0 \mathrm{~mol} / \mathrm{L}($ blue $)$ and $7 \times 10^{-3} \mathrm{~mol} / \mathrm{L}($ black $)$. 


\section{VIII) Microfabrication}

\section{Python script}

A home-made Python script was designed to automatically determine an average value of line widths and heights over a length of several micrometers of the middle of each line. The script proceeds as follows: first, each SEM picture file, such as the one shown in Figure S22a, is converted to a strictly black and white picture by an automatic thresholding operation so that the line on the source image is entirely white and the surrounding becomes black (Figure S22b). The thresholding method used here is Otsu's binarisation. Then, a contour detection function included in the OpenCV library is applied to this modified image to acquire the coordinates of the white pixels (Figure S22c). For control, we can superimpose the shape that has been detected with the original image to ensure that the contour of the line is correct (Figure S22d). Finally, those white pixels can be counted for each row of pixels on the image which allows us to estimate the width of the line along its whole length. The scale bar of each picture is detected and used to convert the number of pixels into a distance measurement. A vertical line anchored to the glass coverslip is shown on Figure S22, but the script is also able to process horizontal lines and suspended lines so that the line height can be evaluated as well.

The error bars correspond to the $95 \%$ confidence interval given by the student's t-distribution. Thus, it provides information on the error error resulting from the measurement of the line widths and heights by SEM. The values were considered without subtracting the thickness of the platinum layer (expected at $10 \mathrm{~nm}$ ).
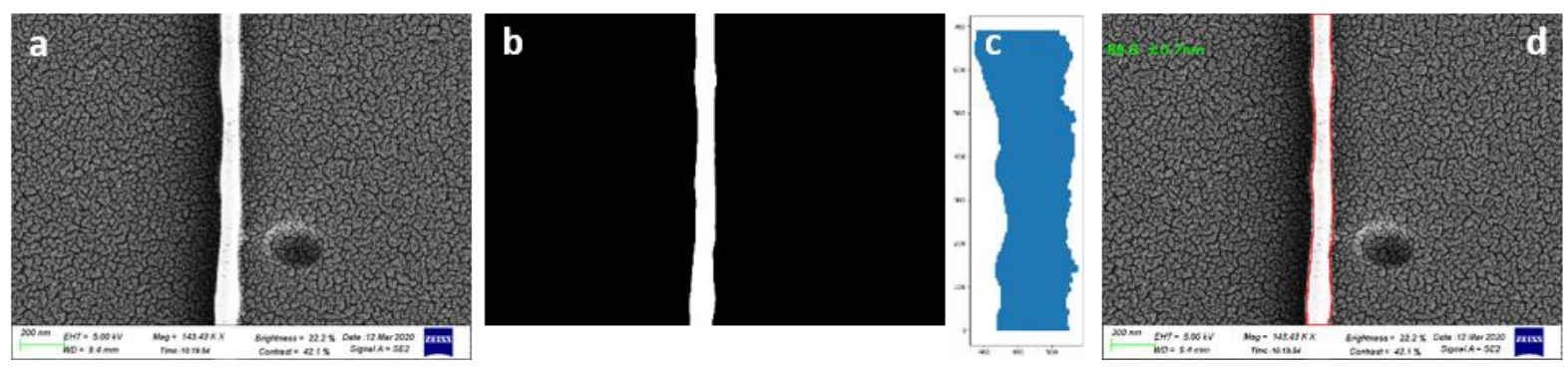

Figure S22. Description of the different steps of the Python script: a) the original image; b) the same image after thresholding; c) the plot of white pixel coordinates; d) the detected contour is shown in red and the average width and the associated error is indicated in green on the final picture.

\section{Evolution of the line widths and heights versus laser power}
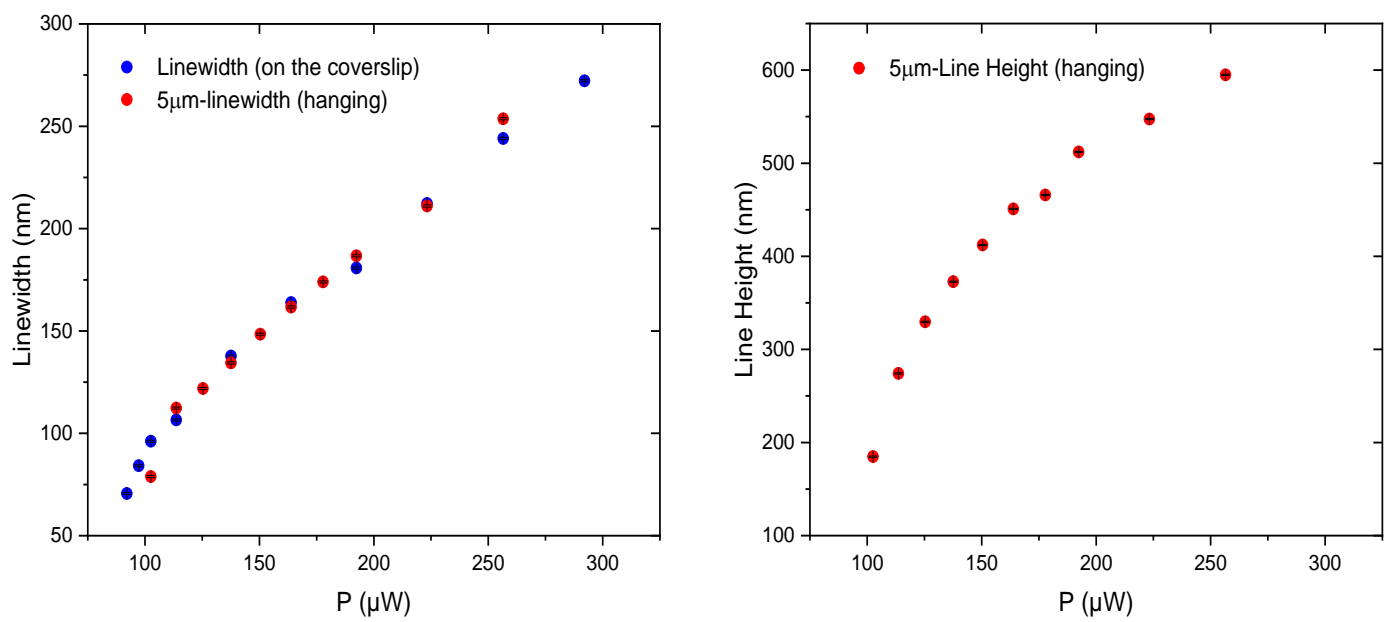

Figure S23. Evolution of the line width (left) and height (right) versus laser power used during the fabrication. The blue dots correspond to structures fabricated at the surface of the coverslip and the red ones correspond to lines suspended between two supports. Writing speed $40 \mu \mathrm{m} / \mathrm{s}$, photoresist: DPPHA:DDA:V-Shape (79.2:19.8:1). 


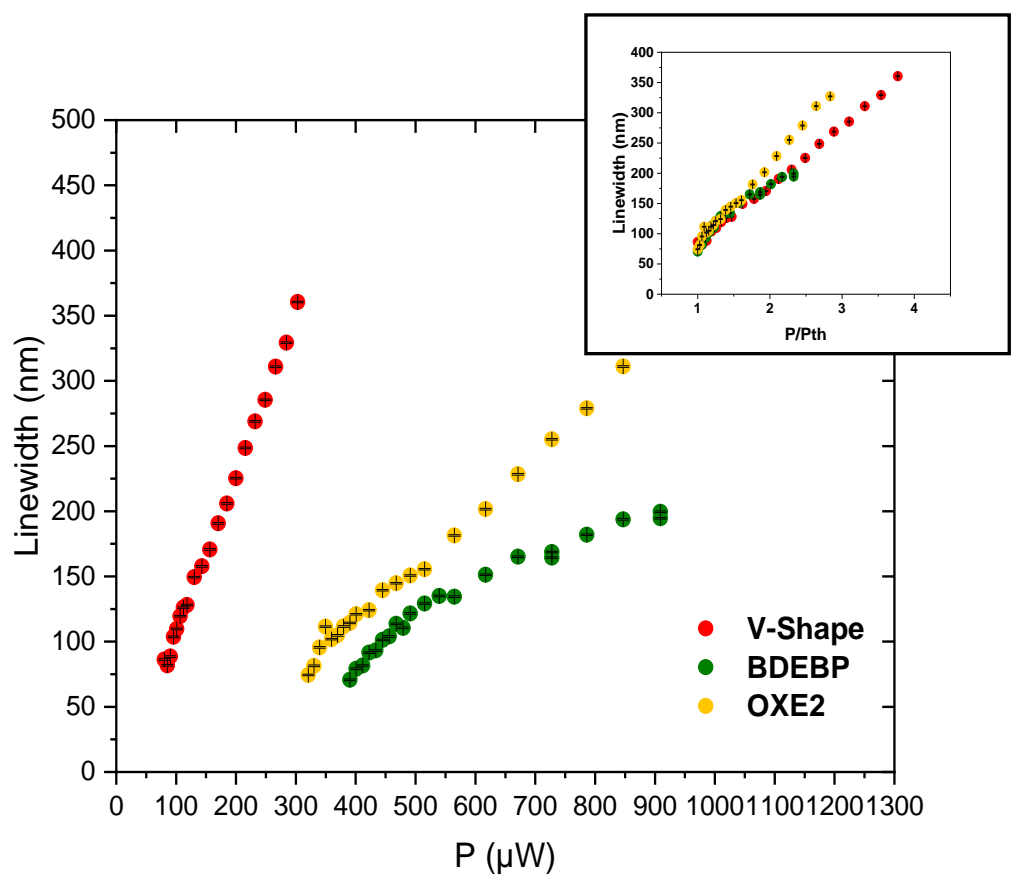

Figure S24. Linewidth versus average laser power (major graph) and ratio average power/threshold power (inset) for three photoresists

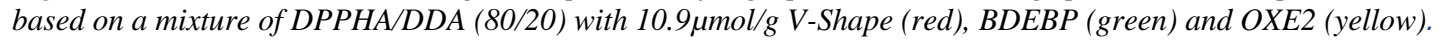


Modeling of the evolution of the line widths and heights

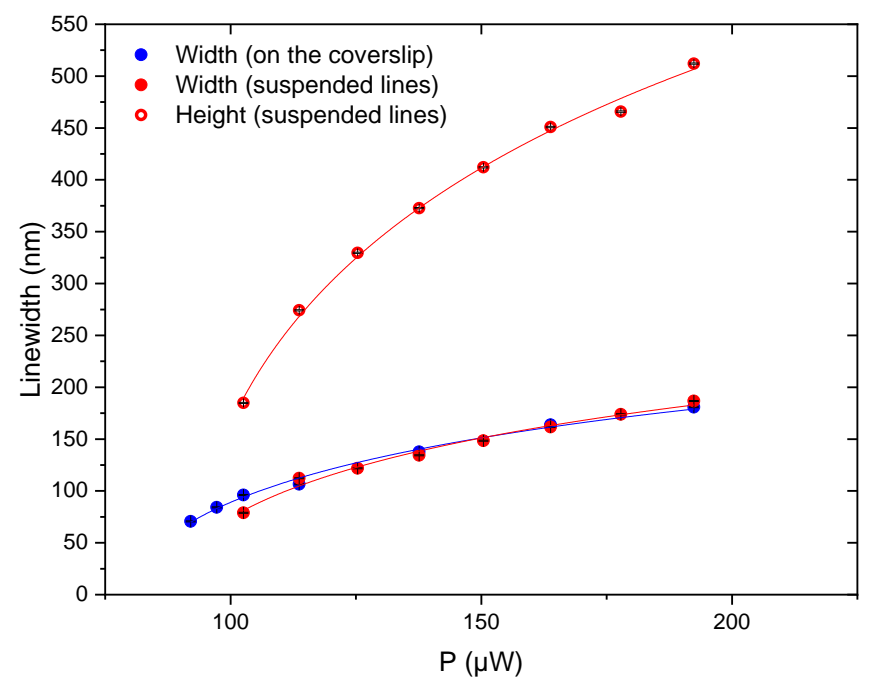

\begin{tabular}{|c|c|c|c|}
\hline \multirow{2}{*}{$\begin{array}{c}\text { Equation } \\
\text { Plot }\end{array}$} & \multicolumn{3}{|c|}{ w.sqrt(2.In(x/Pth)) } \\
\hline & Width (on the coverslip) & Width (suspended) & Height (suspended) \\
\hline$w(n m)$ & $136 \pm 3$ & $146 \pm 3$ & $419 \pm 5$ \\
\hline Pth $(\mu \mathrm{W})$ & $81 \pm 1$ & $88 \pm 2$ & $93 \pm 1$ \\
\hline Adj. $\mathbf{R}^{2}$ & 0.994 & 0.986 & 0.996 \\
\hline
\end{tabular}

Figure S25. Modeling of the evolution of the line width and height versus laser power following equation 3. Writing speed $40 \mu \mathrm{m} / \mathrm{s}$, photoresist: DPPHA:DDA:V-Shape (79.2:19.8:1). $w$ corresponds to $w_{x y}$, the beam waist in the $x$-y plane for the two first columns and to $w_{z}$, the beam waist along the z-axis for the last one.

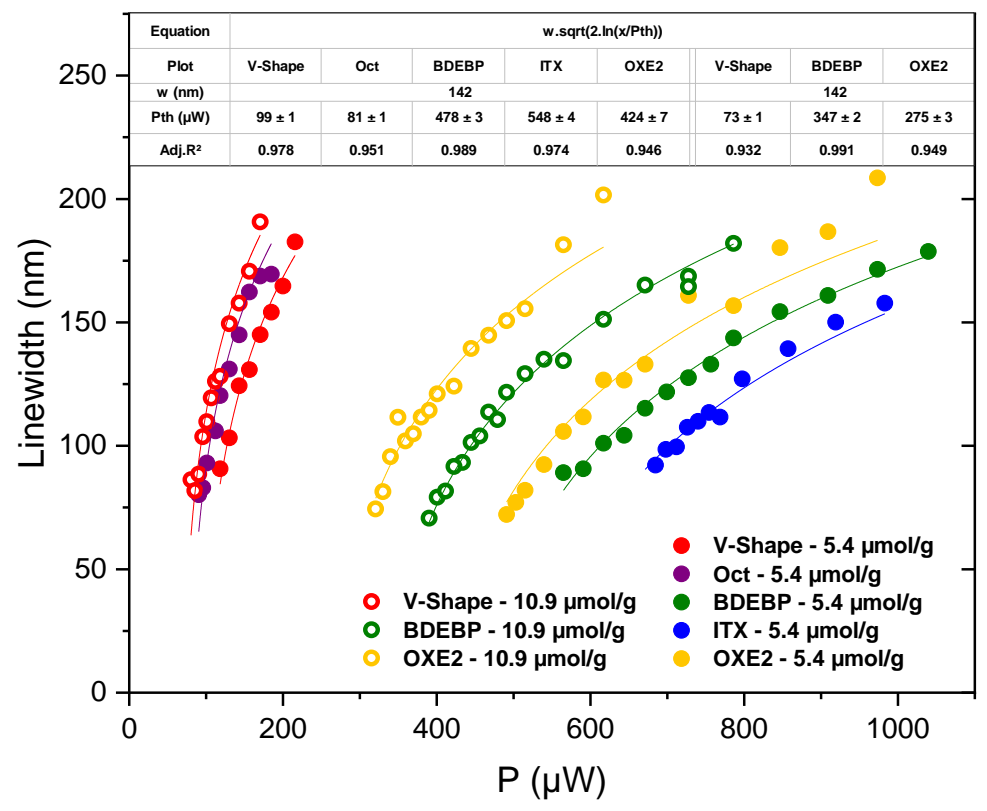

Figure S26. Modeling of the evolution of the line width versus laser power following equation 3. Writing speed $40 \mu \mathrm{m} / \mathrm{s}$. The photoresists are based on a mixture of DPPHA/DDA (80/20) with two different concentrations of the five PIs used in this study. 